\title{
A TWO-FLUID HYPERBOLIC MODEL IN A POROUS MEDIUM
}

\author{
LaËtitia Girault ${ }^{1,2}$ And Jean-Marc HÉrard ${ }^{1}$
}

\begin{abstract}
The paper is devoted to the computation of two-phase flows in a porous medium when applying the two-fluid approach. The basic formulation is presented first, together with the main properties of the model. A few basic analytic solutions are then provided, some of them corresponding to solutions of the one-dimensional Riemann problem. Three distinct Finite-Volume schemes are then introduced. The first two schemes, which rely on the Rusanov scheme, are shown to give wrong approximations in some cases involving sharp porous profiles. The third one, which is an extension of a scheme proposed by Kröner and Thanh [SIAM J. Numer. Anal. 43 (2006) 796-824] for the computation of single phase flows in varying cross section ducts, provides fair results in all situations. Properties of schemes and numerical results are presented. Analytic tests enable to compute the $L^{1}$ norm of the error.
\end{abstract}

Mathematics Subject Classification. 76S05, 76M12, 65M12, 76T10.

Received July 22, 2008. Revised July 20, 2009 and December 18, 2009.

Published online May 10, 2010.

\section{IntRoduction}

The main purpose of the present work is to develop models and schemes that allow the computation of twophase flows in a porous medium while focusing on the two-fluid approach, and thus considering distinct pressure, velocity and temperature fields within each phase. Following the pioneering work of Baer and Nunziatto [4], Kapila et al. [27], and more recent work [9,14], that deals with the two-fluid two-pressure approach in an open medium, we first provide an extension to the framework of porous medium as proposed in [26]. As expected, the system is hyperbolic, at least for small enough Mach number within each phase, which of course seems reasonable when focusing on applications for flows in pressurised water reactors in nuclear power plants. The governing set of equations also benefits from two major features. Though it contains some non-conservative products, all jump conditions are unique in single genuinely non linear - GNL - fields. This is tightly connected with the fact that the closure law for the so-called interfacial velocity is such that the field associated with the eigenvalue $\lambda=V_{I}$ is linearly degenerated - LD (see also [9]). Moreover, we emphasize that a relevant entropy inequality holds for regular solutions of the whole set of equations including source terms (and viscous terms if any).

\footnotetext{
Keywords and phrases. Porous medium, well-balanced scheme, analytic solution, convergence rate, two-phase flow.

${ }^{1}$ EDF, R\&D, Fluid Dynamics, Power Generation and Environment, 6 quai Watier, 78400 Chatou, France.

jean-marc.herard@edf.fr

${ }^{2}$ Centre de Mathématiques et Informatique, LATP, 39 rue Joliot Curie, 13453 Marseille Cedex 13, France.
} 
Once the model is presented (Sect. 2), Section 3 will provide some details on a few analytic solutions of the whole system that will be investigated in Sections 5 and 6 . We will then focus in Section 4 on three simple Finite Volume schemes in order to compute approximations of the latter model in a porous medium. The first scheme corresponds to the classical Rusanov scheme, the second one being a slight modification of the latter. The third scheme is quite different. It relies on former propositions by Greenberg and Leroux (see [21]) revisited by Kröner and Thanh (see [28], and [3,5] too). Actually the latter third scheme does not require solving an exact Riemann problem around each cell interface (see [20]), and thus is much simpler than the original well-balanced scheme [21]. The main properties of the schemes will be given in Section 5, with special emphasis on the well-balanced properties of course, but also on positivity properties. The last two sections will be devoted to the presentation of numerical results. More precisely, Section 6 will give the opportunity to examine the convergence rate of the above mentioned schemes with respect to the mesh size. Eventually, we will focus in Section 7 on the computation of the interaction of moving waves with the standing free/porous interface. Beyond the present work, we would like to mention that we aim at investigating some possible way to ensure a relevant interfacial and unsteady coupling of existing codes associated with free and porous medium respectively. This motivates the numerical experiments that are introduced within the last two sections. For a further insight on the interfacial coupling of models, we refer to the recent work by the working group [1], and more precisely on the early work [18,19], but also to the recent review article [17], and also on [6].

\section{A TWO-FLUID MODEL IN A POROUS MEDIUM}

We first need to present the two-fluid two-pressure model introduced in [26], and we shall also recall some of its properties afterwards.

\subsection{Governing equations for the two-fluid model}

We first introduce the void fraction $\alpha_{k} \in[0,1]$ that complies with $\alpha_{1}+\alpha_{2}=1$, the porosity $\left.\left.\epsilon \in\right] 0,1\right]$, and (for $k=1,2)$ the mean velocity $U_{k}$, the mean pressure $P_{k}$, the mean density $\rho_{k}$, the internal energy $e_{k}=e_{k}\left(P_{k}, \rho_{k}\right)$ in phase $k$. The state variable $W$ in $\mathbf{R}^{8}$ is:

$$
W^{t}=\left(\epsilon, \alpha_{2}, \epsilon m_{1}, \epsilon m_{2}, \epsilon m_{1} U_{1}, \epsilon m_{2} U_{2}, \epsilon E_{1}, \epsilon E_{2}\right) .
$$

We will also use $W_{\epsilon}$ defined as follows:

$$
W_{\epsilon}^{t}=\left(\epsilon m_{k}, \epsilon m_{k} U_{k}, \epsilon E_{k}\right)
$$

in $\mathbf{R}^{6}$, while noting $m_{k}=\alpha_{k} \rho_{k}$ the partial mass in phase $k$, and $E_{k}=m_{k} U_{k}^{2} / 2+m_{k} e_{k}$ the total energy of phase $k$. The equation of state (EOS) is provided through the function $e_{k}\left(P_{k}, \rho_{k}\right)$, which may be arbitrary. We will thus focus herein on the following two-fluid model:

$$
\left\{\begin{array}{l}
\partial_{t}(\epsilon)=0 \\
\partial_{t}\left(\alpha_{2}\right)+V_{I} \partial_{x}\left(\alpha_{2}\right)=\phi_{2}(W) \\
\partial_{t}\left(\epsilon m_{k}\right)+\partial_{x}\left(\epsilon m_{k} U_{k}\right)=0 \\
\partial_{t}\left(\epsilon m_{k} U_{k}\right)+\partial_{x}\left(\epsilon m_{k} U_{k}^{2}\right)+\epsilon \alpha_{k} \partial_{x}\left(P_{k}\right)+\epsilon\left(P_{k}-P_{I}\right) \partial_{x}\left(\alpha_{k}\right)=\epsilon D_{k}(W) \\
\partial_{t}\left(\epsilon E_{k}\right)+\partial_{x}\left(\epsilon U_{k}\left(E_{k}+\alpha_{k} P_{k}\right)\right)+\epsilon P_{I} \partial_{t}\left(\alpha_{k}\right)=\epsilon \psi_{k}+\epsilon V_{I} D_{k}(W)
\end{array}\right.
$$

We now detail the closure laws for the source terms $\left(\phi_{2}, D_{k}, \psi_{k}\right)$, which agree with:

$$
\sum_{k=1}^{2} \psi_{k}(W)=0 ; \quad \sum_{k=1}^{2} D_{k}(W)=0 ; \quad \sum_{k=1}^{2} \phi_{k}(W)=0 .
$$


The latter two read:

$$
\left\{\begin{array}{l}
D_{k}=(-1)^{k} \frac{m_{1} m_{2}}{\left(m_{1}+m_{2}\right)}\left(U_{1}-U_{2}\right) / \tau_{U} \\
\phi_{k}=(-1)^{k} \frac{\alpha_{1} \alpha_{2}}{\left|P_{1}\right|+\left|P_{2}\right|}\left(P_{2}-P_{1}\right) / \tau_{P}
\end{array}\right.
$$

where both $\tau_{U}$ and $\tau_{P}$ denote relaxation time scales. The contribution $D_{k}$ refers to the drag forces. Besides, the energy interfacial transfer term:

$$
\psi_{k}=K_{T}\left(a_{k}-a_{3-k}\right)
$$

requires to define $a_{k}$ :

$$
a_{k}=\left(s_{k}\right)^{-1}\left(\partial_{P_{k}}\left(s_{k}\right)\right)\left(\partial_{P_{k}}\left(e_{k}\right)\right)^{-1}
$$

where $s_{k}=s_{k}\left(P_{k}, \rho_{k}\right)$ denotes the specific entropy, which is compelled with:

$$
\left(c_{k}\right)^{2} \partial_{P_{k}}\left(s_{k}\right)+\partial_{\rho_{k}}\left(s_{k}\right)=0
$$

noting as usual:

$$
\left(c_{k}\right)^{2}=\left(c_{k}\right)^{2}\left(P_{k}, \rho_{k}\right)=\left(\frac{P_{k}}{\left(\rho_{k}\right)^{2}}-\partial_{\rho_{k}}\left(e_{k}\right)\right)\left(\partial_{P_{k}}\left(e_{k}\right)\right)^{-1} .
$$

The couple $\left(V_{I}, P_{I}\right)$ is assumed to be one among the two couples $\left(U_{k}, P_{3-k}\right)$, with $k \in 1,2$. For instance, the pair $\left(U_{2}, P_{1}\right)$ is expected to be physically relevant when the phase 2 is dilute (and reversely the pair $\left(U_{1}, P_{2}\right)$ when the flow is dominated by phase 2). These two pairs correspond to models investigated in $[2,4,27,30,31]$ among others. Note anyway that a third choice corresponding to $\left(V_{m}, P_{m}\right)$ as defined in $[9,14]$ is also meaningful, and might be considered as well.

\subsection{Main properties of the two-fluid model}

First, we focus on the homogeneous part of (2.3), e.g.:

$$
\left\{\begin{array}{l}
\partial_{t}(\epsilon)=0 \\
\partial_{t}\left(\alpha_{2}\right)+V_{I} \partial_{x}\left(\alpha_{2}\right)=0 \\
\partial_{t}\left(\epsilon m_{k}\right)+\partial_{x}\left(\epsilon m_{k} U_{k}\right)=0 \\
\partial_{t}\left(\epsilon m_{k} U_{k}\right)+\partial_{x}\left(\epsilon m_{k} U_{k}^{2}\right)+\epsilon \alpha_{k} \partial_{x}\left(P_{k}\right)+\epsilon\left(P_{k}-P_{I}\right) \partial_{x}\left(\alpha_{k}\right)=0 \\
\partial_{t}\left(\epsilon E_{k}\right)+\partial_{x}\left(\epsilon U_{k}\left(E_{k}+\alpha_{k} P_{k}\right)\right)+\epsilon P_{I} \partial_{t}\left(\alpha_{k}\right)=0
\end{array}\right.
$$

Property 1 (structure of the convective part of (2.3)). The homogeneous system (2.10) admits the following real eigenvalues:

$$
\begin{aligned}
& \lambda_{0}=0, \quad \lambda_{1}=V_{I}, \\
& \lambda_{2}=U_{1}, \quad \lambda_{3}=U_{1}-c_{1}, \quad \lambda_{4}=U_{1}+c_{1}, \\
& \lambda_{5}=U_{2}, \quad \lambda_{6}=U_{2}-c_{2}, \quad \lambda_{7}=U_{2}+c_{2} .
\end{aligned}
$$

Associated right eigenvectors span the whole space if: $\left|V_{I}-U_{k}\right| \neq c_{k}$, and $\left|U_{k}\right| \neq c_{k}$, for $k=1,2$. Otherwise, a resonance phenomenon occurs. Fields associated with eigenvalues $\lambda_{0}, \lambda_{2}, \lambda_{5}$ are linearly degenerated (LD), whereas fields associated with $\lambda_{3}, \lambda_{4}, \lambda_{6}, \lambda_{7}$ are genuinely non-linear. Owing to the particular choice $V_{I}=U_{k}$, the field associated with $\lambda_{1}$ is also $L D$.

For nuclear applications with a mixture of water and vapor, resonance is very unlikely to happen, since material velocities are indeed small compared with the speed of acoustic waves in pure phases. For a more detailed investigation of resonance phenomena, we refer for instance to [16] and also to [8] which focuses on shallow-water equations with topography. 
Property 2 (entropy inequality). Define the entropy-entropy flux pair $\left(\eta, f_{\eta}\right)$ as:

$$
\begin{gathered}
\eta=\epsilon\left(m_{1} \log \left(s_{1}\right)+m_{2} \log \left(s_{2}\right)\right) \\
f_{\eta}=\epsilon\left(m_{1} \log \left(s_{1}\right) U_{1}+m_{2} \log \left(s_{2}\right) U_{2}\right)
\end{gathered}
$$

and the following quantity:

$$
m_{k} R_{k}=a_{k}\left(\psi_{k}+D_{k}\left(V_{I}-U_{k}\right)-\phi_{k}\left(P_{I}-P_{k}\right)\right) .
$$

Then smooth solutions of system (2.3) comply with the following entropy inequality:

$$
\partial_{t}(\eta)+\partial_{x}\left(f_{\eta}\right)=\epsilon\left(m_{1} R_{1}+m_{2} R_{2}\right) \geq 0 \text {. }
$$

Before going further on, it may be noticed that slightly different choices of $P_{I}$ might be considered (see [9,14, $26]$ ), which result in a dissipative contribution in the governing equation of the entropy $\eta$. We insist that these are not considered in the present paper.

Property 3 (Riemann invariants in the standing wave). The linearly degenerated (LD) wave associated with $\lambda=0$ admits the following Riemann invariants

$$
\begin{aligned}
& I_{1}^{0}(W)=\alpha_{2} ; \quad I_{2}^{0}(W)=s_{1} ; \quad I_{3}^{0}(W)=\epsilon m_{1} U_{1} \\
& I_{4}^{0}(W)=e_{1}+\frac{P_{1}}{\rho_{1}}+\frac{U_{1}^{2}}{2} ; \quad I_{5}^{0}(W)=s_{2} ; \\
& I_{6}^{0}(W)=\epsilon m_{2} U_{2} ; \quad I_{7}^{0}(W)=e_{2}+\frac{P_{2}}{\rho_{2}}+\frac{U_{2}^{2}}{2} .
\end{aligned}
$$

The latter Riemann invariants will be used in practice in order to define the third scheme in Section 4.

Property 4 (Riemann invariants in the $V_{I}$ contact discontinuity). We still assume that $V_{I}=U_{k}$. As a consequence, the wave associated with the eigenvalue $\lambda=V_{I}$ is linearly degenerated. Moreover, associated Riemann invariants are the following:

$$
\begin{aligned}
& I_{1}^{1}(W)=\epsilon ; \quad I_{2}^{1}(W)=s_{3-k} ; \\
& I_{3}^{1}(W)=U_{k} ; \quad I_{4}^{1}(W)=m_{3-k}\left(U_{3-k}-U_{k}\right) ; \\
& I_{5}^{1}(W)=\alpha_{1} P_{1}+\alpha_{2} P_{2}+m_{3-k}\left(U_{3-k}-U_{k}\right)^{2} ; \\
& I_{6}^{1}(W)=e_{3-k}+\frac{P_{3-k}}{\rho_{3-k}}+\frac{1}{2}\left(U_{3-k}-U_{k}\right)^{2} .
\end{aligned}
$$

The latter property is obviously extremely important. Actually, even in a free medium, thus corresponding to the uniform distribution $\epsilon=1$, the specific closure law for the so-called interfacial velocity-pressure pair $\left(V_{I}, P_{I}\right)$ guarantees that the non-conservative products are only active in a linearly degenerated field. Thus unique jump conditions hold field by field, which results in the crucial point that the converged approximations (w.r.t. the mesh size) obtained when computing flows with shock waves through system (2.3) will not depend on the scheme (see [22]), as may happen for other unsuitable choices of $\left(V_{I}, P_{I}\right)$.

\section{BASIC SOLUTIONS}

\subsection{Two simple solutions}

We define two basic solutions of system (2.3), whatever the EOS is. 
- Basic solution $S_{1}$ :

We define solution $S_{1}$ as the following unsteady solution:

$$
\left\{\begin{array}{l}
\epsilon(x)=\epsilon_{0} \\
P_{1}(x, t)=P_{2}(x, t)=P_{0} \\
U_{1}(x, t)=U_{2}(x, t)=U_{0}
\end{array}\right.
$$

while both $\rho_{k}$ and $\alpha_{2}$ are solutions of the governing equation:

$$
\partial_{t}(f)+U_{0} \partial_{x}(f)=0 .
$$

Note that this solution, which is only valid in a free medium, may be viewed as a solution of the sole convective part of system (2.3), or alternatively of the full set of equations (2.3).

- Basic solution $S_{2}$ :

We assume that the distribution $\epsilon(x)$ is arbitrary. Solution $S_{2}$ will correspond to the steady solution:

$$
\left\{\begin{array}{l}
P_{1}(x, t)=P_{2}(x, t)=P_{0} \\
U_{1}(x, t)=U_{2}(x, t)=0
\end{array}\right.
$$

while both $m_{k}(x, t)=m_{k}(x, 0)$ and $\alpha_{2}(x, t)=\alpha_{2}(x, 0)$.

These two basic solutions will be used in order to define a priori suitable schemes. The second solution $S_{2}$ will also be used as a preliminary test case (Test 2) in numerical experiments in Section 6.

\subsection{Solutions of the Riemann problem}

We focus here the homogeneous part of system (2.3), and thus consider now solutions of the Riemann problem associated with system (2.10). We must insist here that we do not know whether there exists a unique solution to the one dimensional Riemann problem for our problem, given left and right initial states. However, we may proceed differently and construct exact solutions. For that purpose we simply introduce a left initial condition, and then construct intermediate states and associated single waves, choosing an initial configuration. We shall restrict here to rather simple choices involving "ghost" waves - through which no variation of the state variable occurs -, and: (i) a steady contact discontinuity, and/or (ii) a moving $V_{I}$ contact discontinuity and/or (iii) a shock wave in phase 2. Solutions will be referred to as Test 1, Test 3 and Test 4 respectively. Of course much more complex test cases might be defined that way, such as those examined in $[2,30,31]$ for instance, but we emphasize here once more that we want to focus on situations where the porosity varies, which explains our choices. The exact construction of intermediate states and the final right state is detailed in Appendix A. Numerical values that are used in numerical experiments are recalled at the beginning of Section 6 , which is devoted to the measure of the $L^{1}$ norm of the error.

Figure 1 provides a sketch of the solution of Riemann problems that will be investigated, together with notations.

\section{Finite Volume SCHEMES}

We introduce standard notations for Finite Volume schemes (see [10]). Within each Finite Volume of size $h_{i}=x_{i+1 / 2}-x_{i-1 / 2}$, the mean value of $W$ at time $t^{n}$ in cell $i$ is:

$$
W_{i}^{n}=\left(\int_{\left[x_{i-1 / 2}, x_{i+1 / 2}\right]} W\left(x, t^{n}\right) \mathrm{d} x\right) / h_{i} .
$$

The time step $\Delta t^{n}$ will comply with a standard CFL condition. Moreover, we define:

$$
\bar{a}_{i+1 / 2}=\left(a_{i}+a_{i+1}\right) / 2
$$




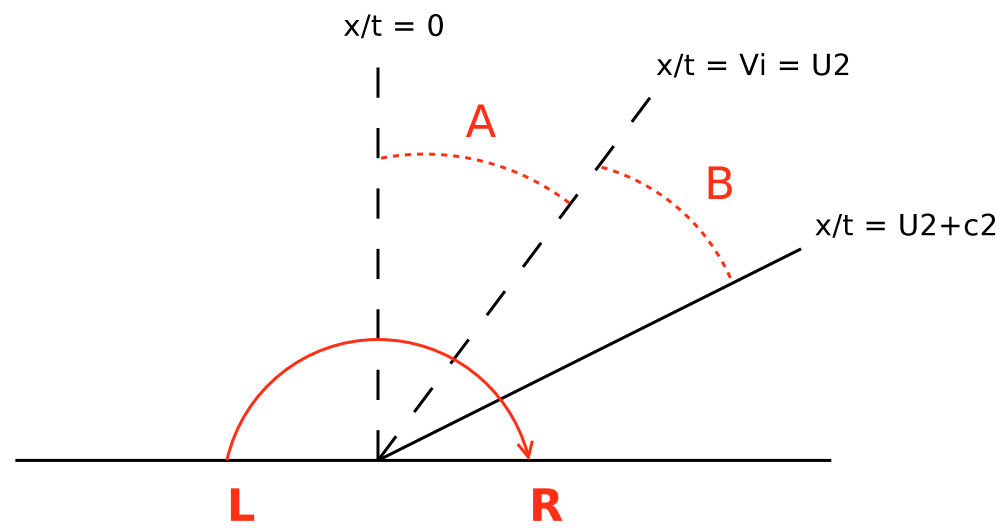

FIGURE 1. Sketch of the specific fan of waves in exact solutions and notations for intermediate states.

whatever the quantity $a$ is, and also:

$$
(\Delta(\bar{a}))_{i}^{n}=(\bar{a})_{i+1 / 2}^{n}-(\bar{a})_{i-1 / 2}^{n}
$$

for $k=1,2$.

We define the flux $f_{\epsilon}$ in $\mathbf{R}^{6}$ :

$$
f_{\epsilon}\left(W_{\epsilon}, \alpha_{2}, \epsilon\right)^{t}=\left(\epsilon m_{k} U_{k}, \epsilon m_{k} U_{k}^{2}, \epsilon U_{k}\left(E_{k}+\alpha_{k} P_{k}\right)\right) .
$$

The computation of the whole set (2.3) is achieved with a fractional step method which is in agreement with the overall entropy inequality. The homogeneous problem associated with (2.10) is computed first. Source terms are then accounted for using an implicit scheme, which is exactly the one described in [14]. We thus only describe the first evolution step here.

\subsection{Classical Rusanov scheme $\boldsymbol{R}$}

The cell scheme which is used to compute the evolution step simply reads:

$$
h_{i}\left(\left(\alpha_{2}\right)_{i}^{n+1}-\left(\alpha_{2}\right)_{i}^{n}\right)+\Delta t^{n}\left(V_{I}\right)_{i}^{n}\left(\Delta\left(\bar{\alpha}_{2}\right)\right)_{i}^{n}+\Delta t^{n}\left(c_{i+1 / 2}^{n}-c_{i-1 / 2}^{n}\right)=0
$$

where $c_{i+1 / 2}^{n}=-r_{i+1 / 2}^{n}\left(\left(\alpha_{2}\right)_{i+1}^{n}-\left(\alpha_{2}\right)_{i}^{n}\right) / 2$, while:

$$
h_{i}\left(\left(W_{\epsilon}\right)_{i}^{n+1}-\left(W_{\epsilon}\right)_{i}^{n}\right)+\Delta t^{n}\left(F_{i+1 / 2}^{R}\left(\left(W_{\epsilon}\right)_{l}^{n},\left(\alpha_{2}\right)_{l}^{n}, \epsilon_{l}\right)-F_{i-1 / 2}^{R}\left(\left(W_{\epsilon}\right)_{l}^{n},\left(\alpha_{2}\right)_{l}^{n}, \epsilon_{l}\right)\right)+\Delta t^{n}\left(H_{\epsilon}\right)_{i}^{n}=0
$$

where the numerical flux is defined by:

$$
F_{i+1 / 2}^{R}\left(\left(W_{\epsilon}\right)_{l}^{n},\left(\alpha_{2}\right)_{l}^{n}, \epsilon_{l}\right)=\left(f_{\epsilon}\left(\left(W_{\epsilon}\right)_{i}^{n},\left(\alpha_{2}\right)_{i}^{n}, \epsilon_{i}\right)+f_{\epsilon}\left(\left(W_{\epsilon}\right)_{i+1}^{n},\left(\alpha_{2}\right)_{i+1}^{n}, \epsilon_{i+1}\right)-r_{i+1 / 2}^{n}\left(\left(W_{\epsilon}\right)_{i+1}^{n}-\left(W_{\epsilon}\right)_{i}^{n}\right)\right) / 2 .
$$

The notation $\left(W_{\epsilon}\right)_{l}^{n}$ involves the stencil 1 that refers to cell indices $(i, i+1)$ for $F_{i+1 / 2}$ (respectively to $(i-1, i)$ for $\left.F_{i-1 / 2}\right)$. The scalar $r_{i+1 / 2}^{n}$ represents the maximal value of the spectral radius of the Jacobian matrices $A\left(\left(W_{\epsilon}\right)_{l}^{n},\left(\alpha_{2}\right)_{l}^{n}, \epsilon_{l}\right)$ for $l=i, i+1$. The contribution connected with the first-order non-conservative terms $\left(H_{\epsilon}\right)_{i}^{n}$ is approximated by:

$$
\left(H_{\epsilon}\right)_{i}^{n}=\left(0, \epsilon_{i}\left(\left(\left(P_{k}\right)_{i}^{n}-\left(P_{I}\right)_{i}^{n}\right)\left(\Delta\left(\bar{\alpha}_{k}\right)\right)_{i}^{n}+\left(\alpha_{k}\right)_{i}^{n}\left(\Delta\left(\bar{P}_{k}\right)\right)_{i}^{n}\right),-\epsilon_{i}\left(P_{I}\right)_{i}^{n}\left(V_{I}\right)_{i}^{n}\left(\Delta\left(\bar{\alpha}_{k}\right)\right)_{i}^{n}\right) .
$$




\subsection{A modified Rusanov scheme $M R$}

This scheme is similar to the previous one. Its main interest is that it guarantees that the steady solution $S_{2}$ will be perfectly approximated on any mesh size (see Sect. 5 below).

The update for the void fractions is:

$$
h_{i}\left(\left(\alpha_{2}\right)_{i}^{n+1}-\left(\alpha_{2}\right)_{i}^{n}\right)+\Delta t^{n}\left(V_{I}\right)_{i}^{n}\left(\Delta\left(\bar{\alpha}_{2}\right)\right)_{i}^{n}+\Delta t^{n}\left(d_{i+1 / 2,-}^{n}-d_{i-1 / 2,+}^{n}\right)=0
$$

where:

$$
\begin{aligned}
& d_{i+1 / 2,-}^{n}=-(\hat{\epsilon})_{i+1 / 2} r_{i+1 / 2}^{n}\left(\left(\alpha_{2}\right)_{i+1}^{n}-\left(\alpha_{2}\right)_{i}^{n}\right) /\left(2 \epsilon_{i}\right) \\
& d_{i-1 / 2,+}^{n}=-(\hat{\epsilon})_{i-1 / 2} r_{i-1 / 2}^{n}\left(\left(\alpha_{2}\right)_{i}^{n}-\left(\alpha_{2}\right)_{i-1}^{n}\right) /\left(2 \epsilon_{i}\right) .
\end{aligned}
$$

The numerical flux in (4.4) is replaced by:

$$
F_{i+1 / 2}^{M R}\left(\left(W_{\epsilon}\right)_{l}^{n},\left(\alpha_{2}\right)_{l}^{n}, \epsilon_{l}\right)=\left(f_{\epsilon}\left(\left(W_{\epsilon}\right)_{i}^{n},\left(\alpha_{2}\right)_{i}^{n}, \epsilon_{i}\right)+f_{\epsilon}\left(\left(W_{\epsilon}\right)_{i+1}^{n},\left(\alpha_{2}\right)_{i+1}^{n}, \epsilon_{i+1}\right)-r_{i+1 / 2}^{n}(\hat{\epsilon})_{i+1 / 2}\left(\frac{\left(W_{\epsilon}\right)_{i+1}^{n}}{\epsilon_{i+1}}-\frac{\left(W_{\epsilon}\right)_{i}^{n}}{\epsilon_{i}}\right)\right) / 2
$$

where $(\hat{\epsilon})_{i+1 / 2}=\max \left(\epsilon_{i}, \epsilon_{i+1}\right)$, or: $(\hat{\epsilon})_{i+1 / 2}=\left(2 \epsilon_{i} \epsilon_{i+1}\right) /\left(\epsilon_{i}+\epsilon_{i+1}\right)$.

\subsection{A simplified well-balanced scheme $W B R$}

The basic idea is the following. For the sake of simplicity, we introduce $Z \in \mathbf{R}^{7}$ and $f(Z) \in \mathbf{R}^{7}$ as follows:

$$
\begin{aligned}
& Z^{t}=\left(\alpha_{2}, m_{k}, m_{k} U_{k}, E_{k}\right) \\
& f(Z)^{t}=\left(0, m_{k} U_{k}, m_{k} U_{k}^{2}+\alpha_{k} P_{k}, U_{k}\left(E_{k}+\alpha_{k} P_{k}\right)\right) .
\end{aligned}
$$

Now, since $\epsilon$ is assumed to be constant within each cell, the cell scheme will read:

$$
h_{i}\left(Z_{i}^{n+1}-Z_{i}^{n}\right)+\Delta t^{n}\left(F_{i+1 / 2,-}^{W B R}\left(Z_{l}^{n}, \epsilon_{l}\right)-F_{i-1 / 2,+}^{W B R}\left(Z_{l}^{n}, \epsilon_{l}\right)\right)+\Delta t^{n} H_{i}^{n}=0
$$

where the numerical fluxes and the contribution $H$ are defined by:

$$
\begin{gathered}
F_{i+1 / 2,-}^{W B R}\left(Z_{l}^{n}, \epsilon_{l}\right)=\left(f\left(Z_{i}^{n}\right)+f\left(Z_{i+1 / 2,-}^{n}\right)-\left(r_{W B}\right)_{i+1 / 2}^{n}\left(Z_{i+1 / 2,-}^{n}-Z_{i}^{n}\right)\right) / 2 \\
F_{i-1 / 2,+}^{W B R}\left(Z_{l}^{n}, \epsilon_{l}\right)=\left(f\left(Z_{i}^{n}\right)+f\left(Z_{i-1 / 2,+}^{n}\right)-\left(r_{W B}\right)_{i-1 / 2}^{n}\left(Z_{i}^{n}-Z_{i-1 / 2,+}^{n}\right)\right) / 2 \\
H_{i}^{n}=\left(\left(V_{I}\right)_{i}^{n}\left(\Delta\left(\bar{\alpha}_{2}\right)\right)_{i}^{n}, 0,-\left(P_{I}\right)_{i}^{n}\left(\Delta\left(\bar{\alpha}_{k}\right)\right)_{i}^{n},-\left(P_{I}\right)_{i}^{n}\left(V_{I}\right)_{i}^{n}\left(\Delta\left(\bar{\alpha}_{k}\right)\right)_{i}^{n}\right) .
\end{gathered}
$$

The values $Z_{i-1 / 2,+}^{n}$ and $Z_{i+1 / 2,-}^{n}$ are obtained by solving the non-linear equations (for $m=0$ to 6 ):

$$
\begin{aligned}
& \operatorname{Inv}_{m}^{0}\left(Z_{i-1 / 2,+}^{n}, \epsilon_{i}\right)=\operatorname{Inv}_{m}^{0}\left(Z_{i-1}^{n}, \epsilon_{i-1}\right) \\
& \operatorname{Inv}_{m}^{0}\left(Z_{i+1 / 2,-}^{n}, \epsilon_{i}\right)=\operatorname{Inv}_{m}^{0}\left(Z_{i+1}^{n}, \epsilon_{i+1}\right) .
\end{aligned}
$$

In agreement with Section 2 (Property 3), we have set here:

$$
\begin{aligned}
& \operatorname{Inv}_{0}^{0}(Z, \epsilon)=\alpha_{2} \\
& \operatorname{Inv}_{3 k-2}^{0}(Z, \epsilon)=s_{k} \\
& \operatorname{Inv}_{3 k-1}^{0}(Z, \epsilon)=\epsilon m_{k} U_{k} \\
& \operatorname{Inv}_{3 k}^{0}(Z, \epsilon)=e_{k}+P_{k} / \rho_{k}+U_{k}^{2} / 2
\end{aligned}
$$


for $k=1,2$. In practice, this requires solving two uncoupled non-linear scalar equations (one for each phase) at each cell interface $i+1 / 2$, the solution of which is trivial when $\epsilon_{i}=\epsilon_{i+1}$, or when $\left(U_{k}\right)_{i}^{n} \cdot\left(U_{k}\right)_{i+1}^{n}=0$. Details pertaining to the exact solution of the above-mentioned equations are given in Appendix C. We emphasize here that:

$$
\max \left(\left|\left(U_{k}\right)_{i+1 / 2,-}^{n}\right|,\left|\left(U_{k}\right)_{i+1 / 2,+}^{n}\right|, r_{l}^{n}\right)=\left(r_{W B}\right)_{i+1 / 2}^{n} \quad \text { for } \quad k=1,2
$$

where $r_{l}^{n}$ stands for the spectral radius of the Jacobian matrix at time $t^{n}$ for $l=i, i+1$.

We note that the update for $\alpha_{2}$ is exactly the same as the one achieved in (4.3), owing to the specific value of $\operatorname{Inv}_{0}^{0}(Z, \epsilon)$ (which implies that: $\left(\alpha_{2}\right)_{i+1 / 2,-}^{n}=\left(\alpha_{2}\right)_{i+1}^{n}$ and $\left.\left(\alpha_{2}\right)_{i-1 / 2,+}^{n}=\left(\alpha_{2}\right)_{i-1}^{n}\right)$. Obviously when the porosity is uniform $\left(\epsilon_{i-1}=\epsilon_{i}=\epsilon_{i+1}\right)$, this scheme identifies with the standard Rusanov scheme, since $Z_{i-1 / 2,+}^{n}=Z_{i-1}^{n}$ and $Z_{i+1 / 2,-}^{n}=Z_{i+1}^{n}$ in that case, and it also corresponds to scheme $M R$.

\section{MAin PROPERTIES}

We wonder first whether the latter three schemes preserve basic solutions on any mesh, which is of course crucial for industrial applications. For that purpose, we need to introduce some constants $a_{k, 0}$ for both phases, together with two invertible functions $g_{k}(\phi)$. Actually, one may easily check that:

- Property 5. We assume that the EOS takes the form: $\rho_{k} e_{k}\left(P_{k}, \rho_{k}\right)=a_{k, 0} \rho_{k}+g_{k}\left(P_{k}\right)$ in each phase $k$. The three schemes $R, M R$ and $W B R$ described above preserve the discrete form of the basic solution $S_{1}$, whatever the mesh size is, since $\left(U_{1}\right)_{i}^{n}=\left(U_{2}\right)_{i}^{n}=U_{0}$ and $\left(P_{1}\right)_{i}^{n}=\left(P_{2}\right)_{i}^{n}=P_{0}$ imply that $\left(U_{1}\right)_{i}^{n+1}=$ $\left(U_{2}\right)_{i}^{n+1}=U_{0}$ and $\left(P_{1}\right)_{i}^{n+1}=\left(P_{2}\right)_{i}^{n+1}=P_{0}$, if $\epsilon_{i}=\epsilon_{0}$.

The reader is referred to Appendix B, Section B.1, for proof, which is almost obvious. In practice, standard EOS such as perfect gas EOS or stiffened gas EOS belong to the above mentioned class. We recall that the stiffened gas EOS simply stands for $a_{k, 0}=0$ and $g_{k}(\phi)=\left(\phi+\gamma_{k} \Pi_{k}\right) /\left(\gamma_{k}-1\right)$, where constants $\gamma_{k}$ and $\Pi_{k}$ are assumed to be such that: $\gamma_{k}>1$ and $0 \leq \Pi_{k}$.

Of course (see [11] for such a discussion in the framework of homogeneous models), it does not mean a priori that the schemes will - or won't - converge towards correct solutions.

The next property is also useful for practical applications, though it is not sufficient of course. It requires similar assumptions on the form of the EOS.

- Property 6. We assume that the EOS takes the form: $\rho_{k} e_{k}\left(P_{k}, \rho_{k}\right)=a_{k, 0} \rho_{k}+g_{k}\left(P_{k}\right)$ in each phase $k$. Both schemes $M R$ and $W B R$ preserve the discrete form of the basic solution $S_{2}$ on any mesh, since $\left(U_{1}\right)_{i}^{n}=\left(U_{2}\right)_{i}^{n}=0$ and $\left(P_{1}\right)_{i}^{n}=\left(P_{2}\right)_{i}^{n}=P_{0}$ imply that $\left(U_{1}\right)_{i}^{n+1}=\left(U_{2}\right)_{i}^{n+1}=0$ and also $\left(P_{1}\right)_{i}^{n+1}=$ $\left(P_{2}\right)_{i}^{n+1}=P_{0}$, with arbitrary $\epsilon_{i}$. The standard $R$ scheme does not.

A proof is detailed in Appendix B, Section B.2, which is again almost obvious, and only requires a few calculations. The structure of the scheme $M R$ with respect to the void fraction is of course mandatory to ensure the result.

- Property 7. We use notations introduced in Section 2 pertaining to Riemann invariants. If we assume that $\epsilon_{L} \neq \epsilon_{R}$, and also that the initial conditions $\left(W_{L}, W_{R}\right)$ of the Riemann problem comply with: $I_{m}^{0}\left(W_{L}\right)=I_{m}^{0}\left(W_{R}\right)$, for $m=1$ to 7 , we are ensured that the scheme $W B R$ preserves steady states on any mesh. This does not hold true for schemes $R$ and $M R$.

The proof for schemes $R$ and $M R$ is not detailed here since it is obvious. The one pertaining to scheme $W B R$ is given in Appendix B, Section B.3. Actually, it is also close to some results stated in [5]. 
The proof is also almost the same as the one given in [29] in the case of Euler equations with perfect gas EOS, in a one-dimensional framework, where authors examine the particular case of flows in variable cross section ducts. It occurs in fact in the proof that, though the present system is indeed much more complex than the one examined in [29], both phases almost "decouple" through the interface, since the void fraction is one among the seven Riemann invariants of the standing wave associated with $\lambda_{0}$ (see Sect. 2). A straightforward consequence is that the governing equations for the mass, momentum and total energy within phase $k$ in a porous medium almost behave "locally" as the Euler equations in a porous medium.

- Property 8. The maximum principle for the void fractions holds, and positive cell values of partial masses are ensured when applying any scheme among $R, M R$ and $W B R$, provided that the following CFL conditions hold:

$R$ scheme:

$$
\frac{\Delta t^{n}}{2 h_{i}}\left(r_{i+1 / 2}^{n}+r_{i-1 / 2}^{n}\right) \leq 1 \quad \forall i
$$

MR scheme $\left(\right.$ with $\left.(\hat{\epsilon})_{i+1 / 2}=\max \left(\epsilon_{i}, \epsilon_{i+1}\right)\right)$ :

$$
\frac{\Delta t^{n}}{2 h_{i}}\left(\frac{(\hat{\epsilon})_{i+1 / 2}}{\epsilon_{i}} r_{i+1 / 2}^{n}+\frac{(\hat{\epsilon})_{i-1 / 2}}{\epsilon_{i}} r_{i-1 / 2}^{n}\right) \leq 1 \quad \forall i
$$

$W B R$ scheme:

$$
\frac{\Delta t^{n}}{2 h_{i}}\left(\left(r_{W B}\right)_{i+1 / 2}^{n}+\left(r_{W B}\right)_{i-1 / 2}^{n}\right) \leq 1 \quad \forall i
$$

Proofs are given in Appendix B, Section B.4.

These results were expected, owing to the specific structure of the underlying Rusanov scheme. The CFL-like condition is almost classical for both $R$ and $W B R$ schemes, and it is slightly different for the $M R$ scheme.

\section{Convergence Rate for analytic solutions}

We examine in this section the true convergence rate of the above mentioned schemes, when computing Riemann problems as explained in Section 3. We do not present all results for the three schemes in any case, but we concentrate on the main features, drawbacks and advantages of schemes.

For all cases, we use uniform meshes, and the range of the mesh size will be recalled in each case. The coarser mesh contains 100 cells, whereas the finer mesh contains $8 \times 10^{5}$ cells. More precisely, we use:

\begin{tabular}{|c|c|c|c|}
\hline Schemes & $\mathrm{R}$ & MR & WBR \\
\hline Test case 1 & \multicolumn{3}{|c|}{$10^{2}$ to $2 \times 10^{5}$ cells } \\
\hline Test case 2 & $10^{2}$ to $2 \times 10^{5}$ cells & $10^{2}$ to $2 \times 10^{5}$ cells & $10^{2}$ to $2 \times 10^{5}$ cells \\
\hline Test case 3 & $10^{2}$ to $4 \times 10^{5}$ cells & - & - \\
\hline Test case 4 & $10^{2}$ to $4 \times 10^{5}$ cells & $10^{2}$ to $4 \times 10^{5}$ cells & $10^{2}$ to $8 \times 10^{5}$ cells \\
\hline
\end{tabular}

The EOS for the vapor phase $(k=2)$ and the water phase $(k=1)$ are assumed to be perfect gas EOS, and the corresponding constants will be $\gamma_{1}=1.1$ and $\gamma_{2}=1.4$. 
TEST 1: Free medium

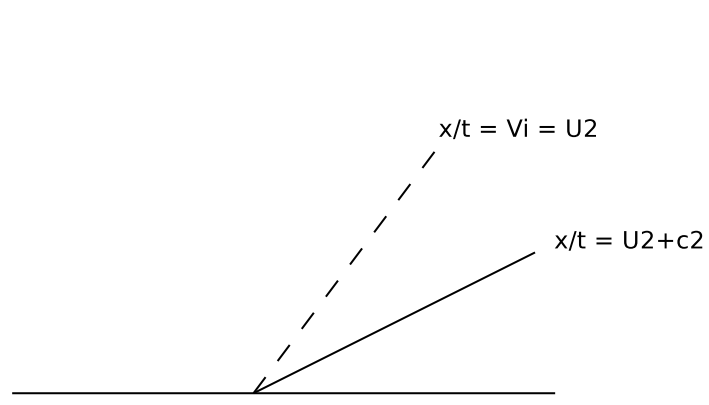

TEST 3: Double contact discontinuity

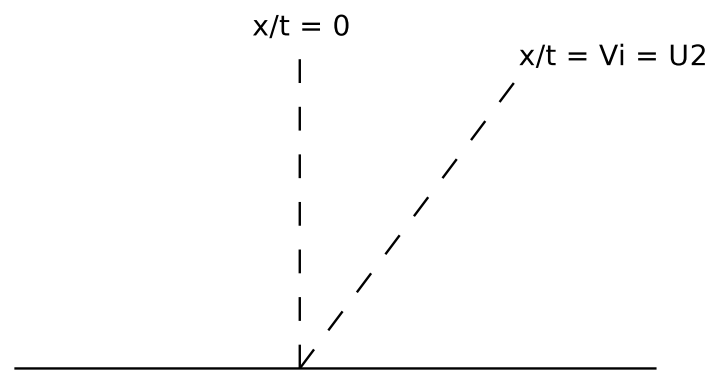

TEST 2: Solution $S_{2}$

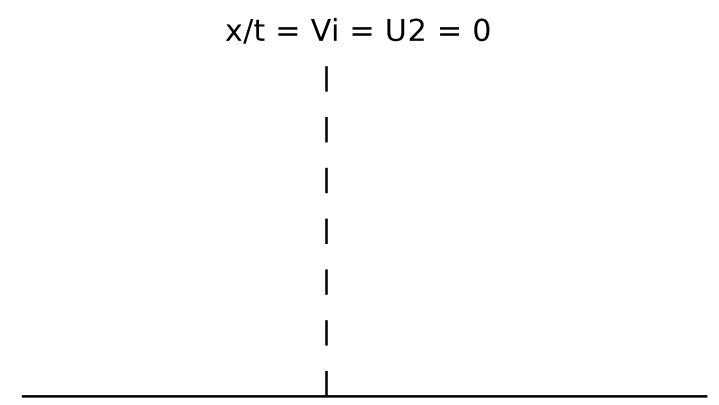

TEST 4: Three-wave pattern

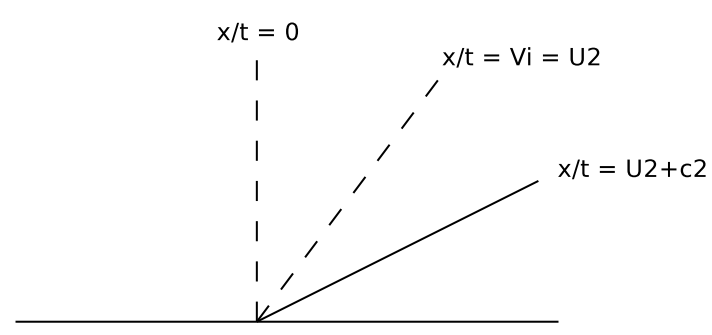

The measure of the $L^{1}$ norm of the error will be provided, together with a "local" estimation of the convergence rate when meaningful. To be more complete, we recall that we use the pair $\left(V_{I}, P_{I}\right)=\left(U_{2}, P_{1}\right)$. In all computations, we use a CFL number $1 / 2$ in order to compute the value $\Delta t^{n}$ at each time step. We also recall below the main configurations that will be investigated, as explained in Section 3.

\subsection{Test case 1: A two-wave Riemann problem in a free medium}

The first solution corresponds to a very simple flow pattern in a free medium. It only involves one void fraction contact-discontinuity (associated with $\lambda=U_{2}$ ), and a shock wave in the vapor phase corresponding to the eigenvalue $\lambda_{7}=U_{2}+c_{2}$. Thus left and right initial states are separated by an intermediate state labeled $B$. We provide below the exact initial data. We recall that results obtained with $R, M R, W B R$ schemes are identical for this test case in a free medium.

\begin{tabular}{|c|c|c|c|}
\hline & State $L$ & State $B$ & State $R$ \\
\hline$\epsilon$ & \multicolumn{3}{|c|}{1} \\
\hline$\alpha_{1}$ & 0.95 & \multicolumn{2}{|c|}{0.05} \\
\hline$\rho_{1}$ & 1 & \multicolumn{2}{|c|}{0.956131034} \\
\hline$U_{1}$ & 10 & \multicolumn{2}{|c|}{-84.3587663} \\
\hline$P_{1}$ & 100000 & \multicolumn{2}{|c|}{95185.1407} \\
\hline$\rho_{2}$ & 0.1 & 0.15 & 0.1 \\
\hline$U_{2}$ & \multicolumn{2}{|r|}{15} & -357.299567 \\
\hline$P_{2}$ & 10000 & 95044.7777 & 53462.6875 \\
\hline
\end{tabular}




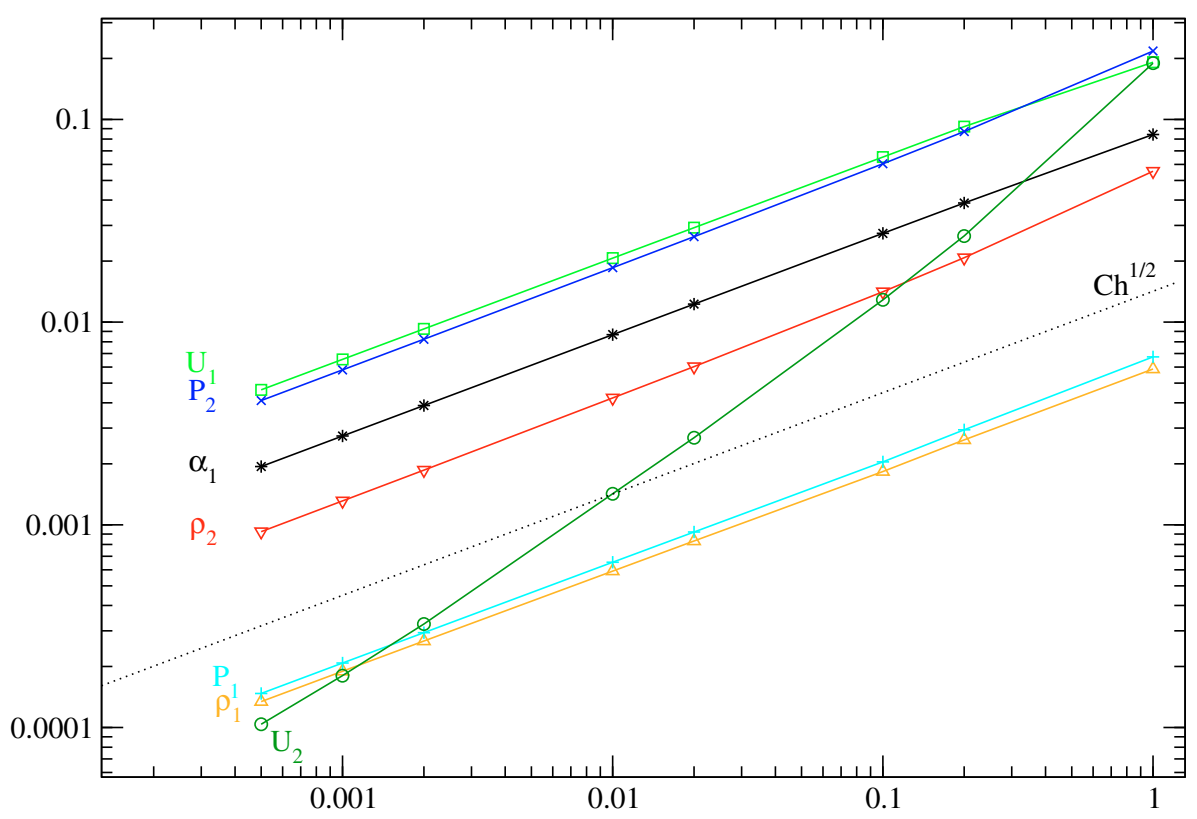

FiguRe 2. $L_{1}$ norm of the error for the $R$ scheme when computing test case 1 (flow in a free medium) as a function of the mesh size. (Figures in color are available online at www . esaim-m2an.org/.)

Computations have been performed using regular meshes with $10^{2}, 5 \times 10^{2}, 10^{3}, 5 \times 10^{3}, 10^{4}, 5 \times 10^{4}, 10^{5}$, and $2 \times 10^{5}$ cells. These enable to plot the $L^{1}$ norm of the error $e_{h}$-in logarithmic coordinates - in Figure 2 .

One may deduce the measure of the rate of convergence $\beta$ at time $t=T$, while focusing on the four finer meshes, and enforcing the behaviour $e_{h}^{\phi}(T)=C_{\phi}(T) h^{\beta(\phi)}$.

\begin{tabular}{|c|c|c|c|}
\hline & Between $10^{4}$ and $5 \times 10^{4}$ cells & Between $5 \times 10^{4}$ and $10^{5}$ cells & Between $10^{5}$ and $2 \times 10^{5}$ cells \\
\hline$\alpha_{1}$ & 0.500 & 0.500 & 0.500 \\
\hline$\rho_{1}$ & 0.494 & 0.496 & 0.497 \\
\hline$U_{1}$ & 0.499 & 0.500 & 0.500 \\
\hline$P_{1}$ & 0.497 & 0.498 & 0.498 \\
\hline$\rho_{2}$ & 0.509 & 0.505 & 0.503 \\
\hline$U_{2}$ & 0.919 & 0.846 & 0.797 \\
\hline$P_{2}$ & 0.505 & 0.503 & 0.502 \\
\hline
\end{tabular}

These values of $\beta(\phi)$ were actually expected. When restricting to $\rho_{1}, U_{1}, P_{1}$, and on $\alpha_{1}$ which only vary in this test case through the void fraction contact discontinuity, an asymptotic rate of 0.5 is "perfect". Moreover, since $\left(\rho_{2}, P_{2}\right)$ vary through both waves, the same is expected. A contrario, the rate $\beta\left(U_{2}\right)$ should be close to 1 since $U_{2}$ is a Riemann invariant through the void fraction contact discontinuity. As a matter of fact, the measured value seems to be close to 0.8 , and this agrees with measurements performed in [12]. 


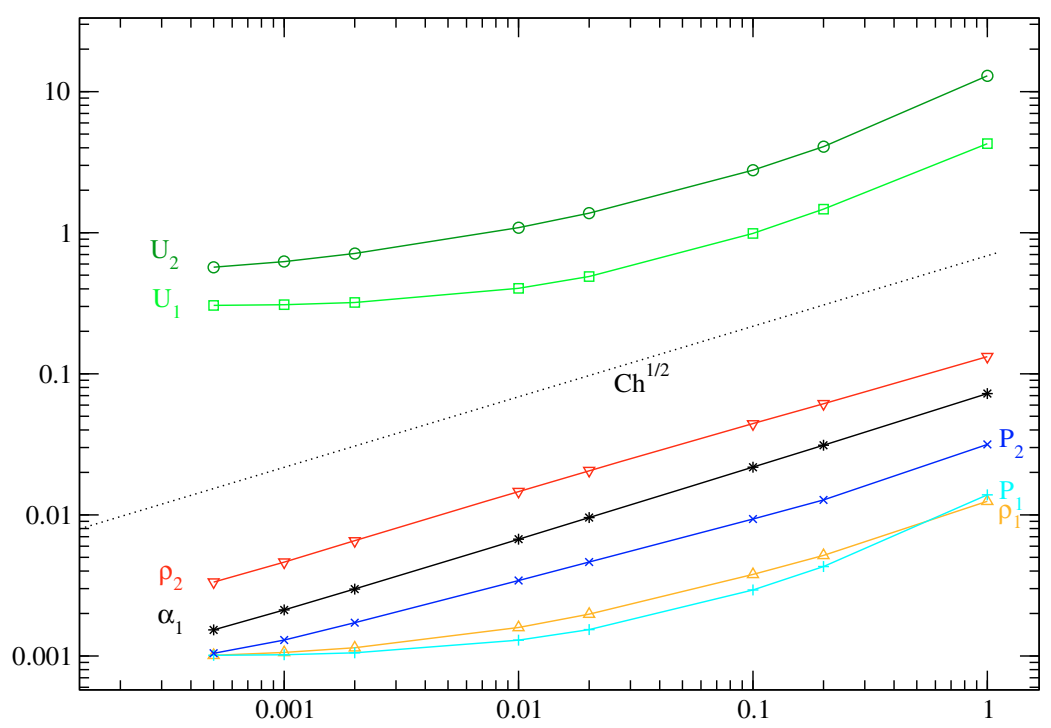

Figure 3. Test 2: $L_{1}$ norm of the error for scheme $R$.

\subsection{Solution $S_{2}$ : A simple steady flow with a free/porous interface}

We now focus on the behaviour of schemes $R, M R$ and $W B R$ when computing approximations of solution labeled $S_{2}$, whose initial data is given below:

\begin{tabular}{|c|c|c|}
\hline & State L & State R \\
\hline$\epsilon$ & 1 & 0.6 \\
\hline$\alpha_{1}$ & 0.95 & 0.05 \\
\hline$\rho_{1}$ & \multicolumn{2}{|c|}{1} \\
\hline$U_{1}$ & \multicolumn{2}{|c|}{0} \\
\hline$P_{1}$ & \multicolumn{2}{|c|}{100000} \\
\hline$\rho_{2}$ & 2 & 0.15 \\
\hline$U_{2}$ & \multicolumn{2}{|c|}{0} \\
\hline$P_{2}$ & \multicolumn{2}{|c|}{100000} \\
\hline
\end{tabular}

Results with $R$ scheme

Simulations involve meshes with $10^{2}, 5 \times 10^{2}, 10^{3}, 5 \times 10^{3}, 10^{4}, 5 \times 10^{4}, 10^{5}$, and $2 \times 10^{5}$ regular cells. Figure 3 displays the $L^{1}$ norm of the error $e_{h}$.

The $R$ scheme clearly no longer converges towards the correct solution when the mesh size goes to zero. The illusion that $\alpha_{1}$ and $\rho_{2}$ still converge is due to the fact that their initial condition is discontinuous, which dissimulates the incorrect behaviour on these "coarse" meshes (wrt to what is sought!). This renders the whole rather dangerous: if we restrict to a range of meshes with 100 up to 10000 cells (the latter represents a "fine mesh" for practical applications...), the $R$ scheme looks almost correct, and one may expect that the pollution will reduce when $h$ tends towards $0 \ldots$ which is obviously not true. Actually, estimations of "convergence rates" $\beta(\phi)$ on the finer meshes are $0.067,0.017,0.014$ for $\rho_{1}, U_{1}$ and $P_{1}$ respectively.

\section{Results with MR scheme}

The meshes are exactly the same. The $L^{1}$ norm of the error $e_{h}$ has been plotted in Figure 4 . Restricting to the finer meshes, approximations of convergence rates $\beta(\phi)$ for $\rho_{2}$ and $\alpha_{1}$ are clearly $1 / 2$. Round-off errors are observed for all other variables. 


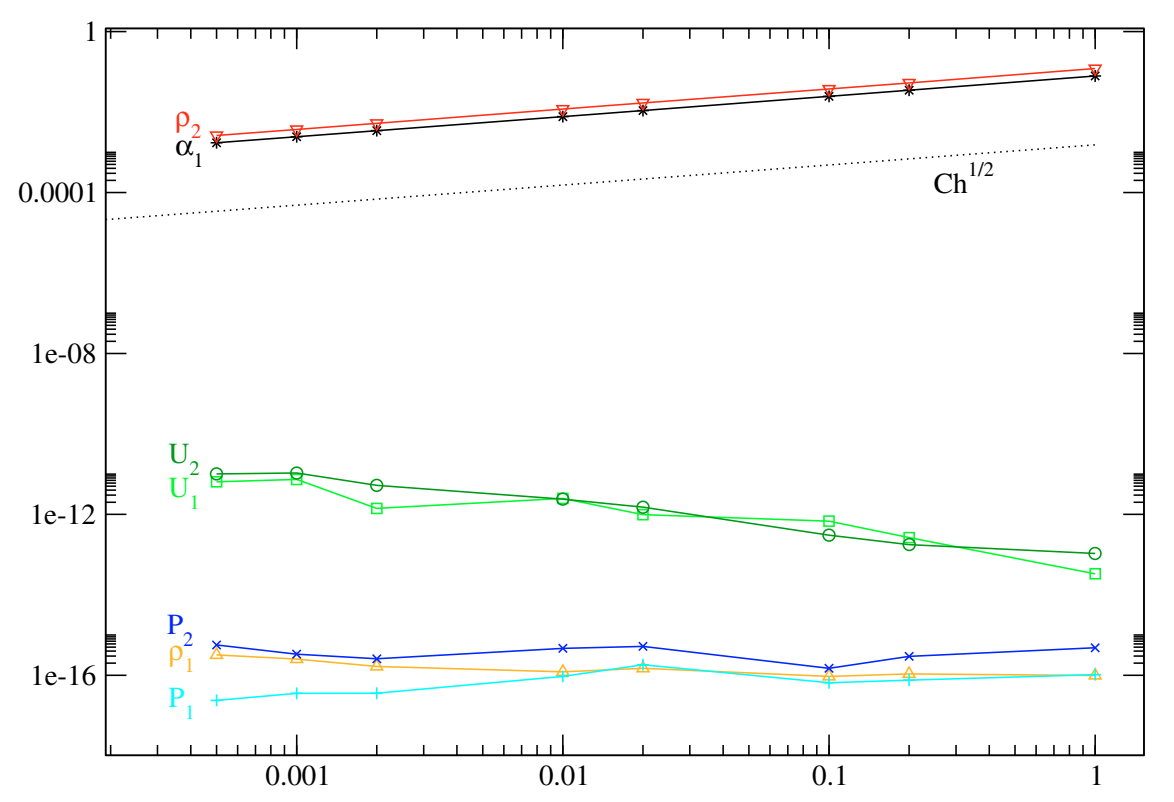

Figure 4. Test 2: $L_{1}$ norm of the error for scheme $M R$.

\section{Results with $W B R$ scheme}

Meshes are still the same, and results are very similar to those obtained with the latter scheme $M R$. The $L^{1}$ norm of the error $e_{h}$ has been plotted in Figure 5. We have also plotted in Figures 6 approximations of both densities $\rho_{1}, \rho_{2}$ that have been obtained using $R, M R, W B R$ schemes on a rather coarse mesh with 1000 nodes. This clearly shows the poor accuracy of the Rusanov scheme: the approximations are spurious around the steady interface, and fast waves propagate on both sides apart from the coupling interface $x=0.5$. This is of course even more astonishing when looking at the density profile in phase 1.

\subsection{Test 3: A combination of a standing contact wave and a void fraction contact discontinuity}

This test case is similar to the second one, but it also involves a contact discontinuity associated with the void fraction wave $\left(\lambda_{5}=U_{2}\right)$. The intermediate state $A$ has been calculated using Appendix A. Meshes now range from $10^{2}$ cells up to $4 \times 10^{5}$ cells. The initial data is as follows:

\begin{tabular}{|c|c|c|c|}
\hline & State $L$ & State $A$ & State $R$ \\
\hline$\epsilon$ & 1 & \multicolumn{2}{|c|}{0.6} \\
\hline$\alpha_{1}$ & \multicolumn{2}{|r|}{0.95} & 0.05 \\
\hline$\rho_{1}$ & 1 & 0.999190167 & 0.853058301 \\
\hline$\overline{U_{1}}$ & 10 & 16.6801748 & -160.919041 \\
\hline$P_{1}$ & 100000 & 99910.922 & 83960.8032 \\
\hline$\rho_{2}$ & 0.1 & 0.0998565629 & 0.15 \\
\hline$\overline{U_{2}}$ & 15 & \multicolumn{2}{|c|}{25.0359108} \\
\hline$\overline{P_{2}}$ & 10000 & 9979.92457 & 94534.4211 \\
\hline
\end{tabular}

The $L^{1}$ norm of the error is plotted in Figure 7 when focusing on scheme $R$. 


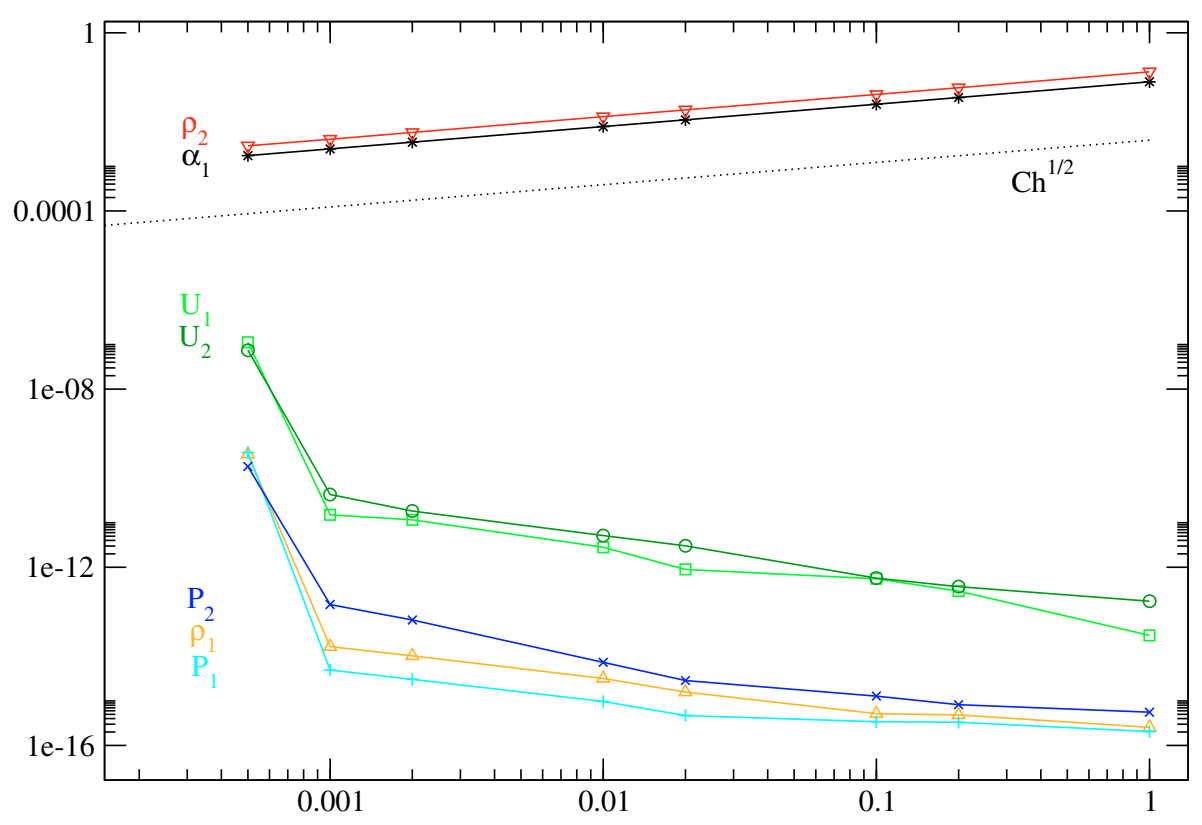

Figure 5. Test 2: $L_{1}$ norm of the error for scheme $W B R$.
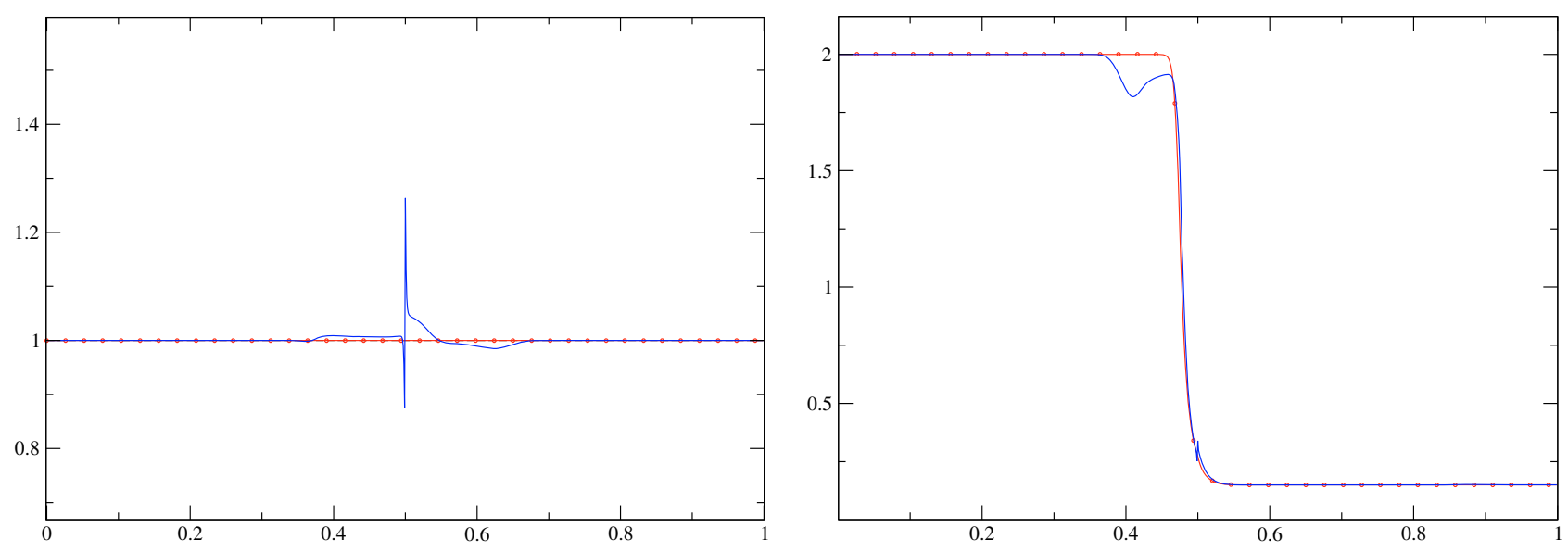

Figure 6 . Test 2: density profiles $\rho_{1}, \rho_{2}$ when using schemes $R$ - blue line,$- M R$ - red line with circles -, WBR - black dotted line - on a 1000-cell mesh.

Once again, we may check that the $R$ scheme no longer converges towards the correct solution, which is of course in agreement with the results of test case 2. We emphasize again that the behaviour on the coarser meshes (on the right side in Fig. 7) is somewhat misleading. 


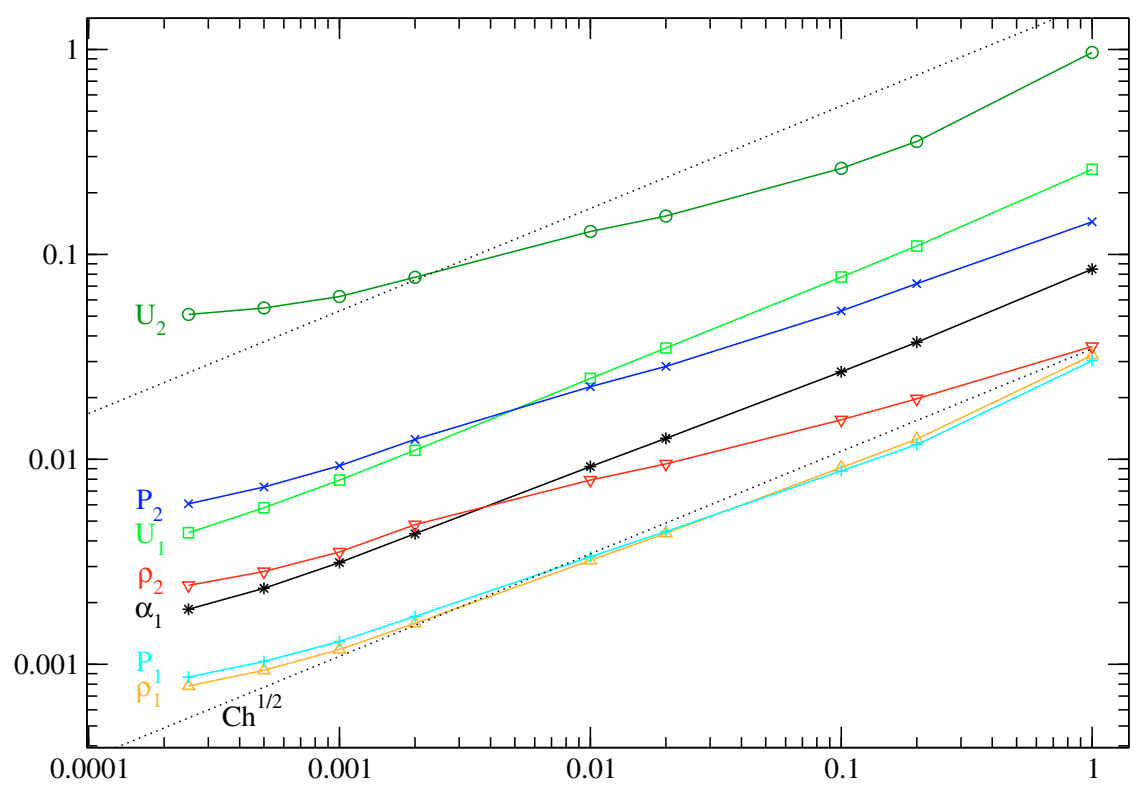

Figure 7 . Test 3: $L_{1}$ norm of the error for scheme $R$.

\subsection{Test 4: A three-wave pattern}

This solution contains two contact waves associated with $\lambda_{0}=0$ and $\lambda_{1}=\lambda_{5}=U_{2}$, and one shock wave in the vapor phase corresponding to $\lambda_{7}=U_{2}+c_{2}$. The exact initial data is given below:

\begin{tabular}{|c|c|c|c|c|}
\hline & State $L$ & State $A$ & State $B$ & State $R$ \\
\hline$\epsilon$ & 1 & \multicolumn{3}{|c|}{0.6} \\
\hline$\alpha_{1}$ & \multicolumn{3}{|c|}{0.95} & \multicolumn{2}{c|}{0.05} \\
\hline$\rho_{1}$ & 1 & 0.999190167 & \multicolumn{2}{|c|}{0.853058301} \\
\hline$U_{1}$ & 10 & 16.6801748 & \multicolumn{2}{|c|}{-160.919041} \\
\hline$P_{1}$ & 100000 & 99910.922 & 83960.8032 \\
\hline$\rho_{2}$ & 0.1 & 0.0998565629 & 0.15 & 0.1 \\
\hline$U_{2}$ & 15 & \multicolumn{2}{|c|}{25.0359108} & -346.262753 \\
\hline$P_{2}$ & 10000 & 9979.92457 & 94534.4211 & 53175.6119 \\
\hline
\end{tabular}

Still using meshes with $10^{2}, 5 \times 10^{2}, 10^{3}, 5 \times 10^{3}, 10^{4}, 5 \times 10^{4}, 10^{5}, 2 \times 10^{5}$ and $4 \times 10^{5}$ cells, we check first that the standard Rusanov scheme does not converge towards the correct solution, and then turn to the $M R$ scheme.

\section{Results with MR scheme}

First we provide in Figure 8 results obtained for $\alpha_{2} \rho_{2}$ when computing the test case on a regular mesh with one thousand cells, together with the exact solution. We still consider meshes used in preceding tests, and errors computed for the $M R$ scheme are given in Figure 9 - on the left side. The crucial point that occurs is that estimations of convergence rates show evidence that the $M R$ scheme no longer converges towards the correct solution. This is quite obvious when focusing on the $U_{2}$ profile in Figure 9 . An approximation of the convergence rate for $U_{2}$ on the finer meshes is 0.035. It obviously means that the "static" Property 6 is far from being sufficient to guarantee convergence of approximations towards the correct solution. 


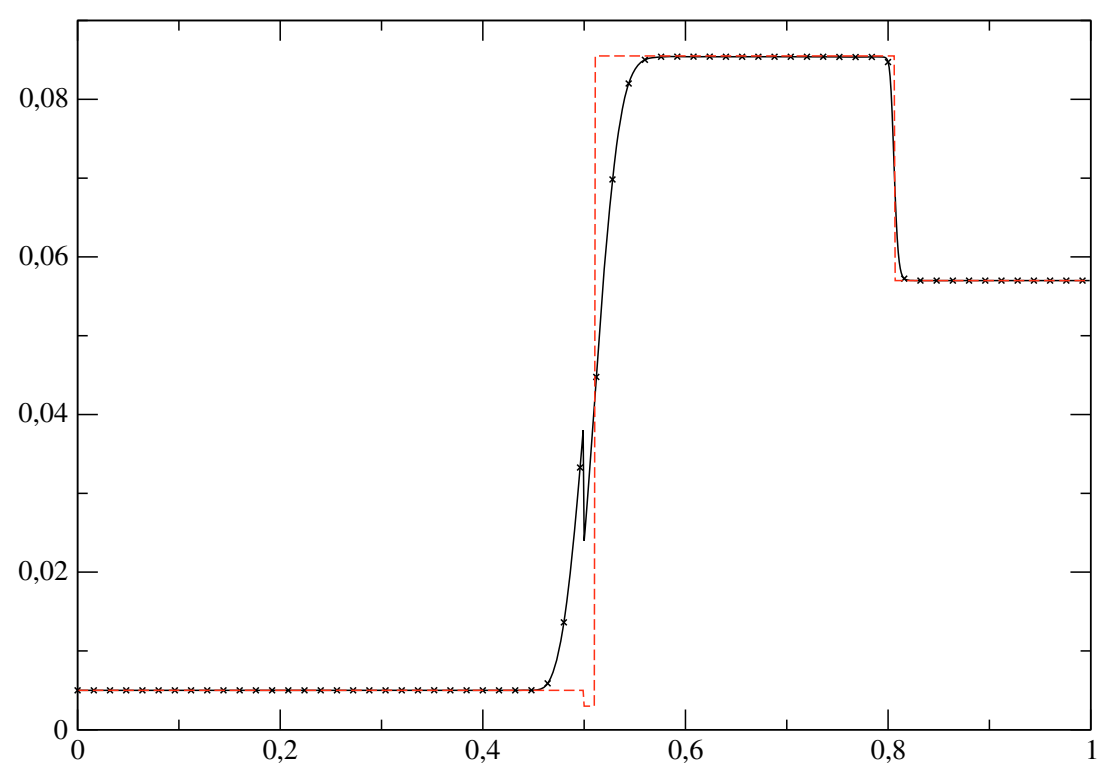

Figure 8. Test 4: mass fraction $\alpha_{2} \rho_{2}$ when using $M R$ scheme - black line with crosses - on a 1000-cell mesh, together with the exact solution - red dashed line.
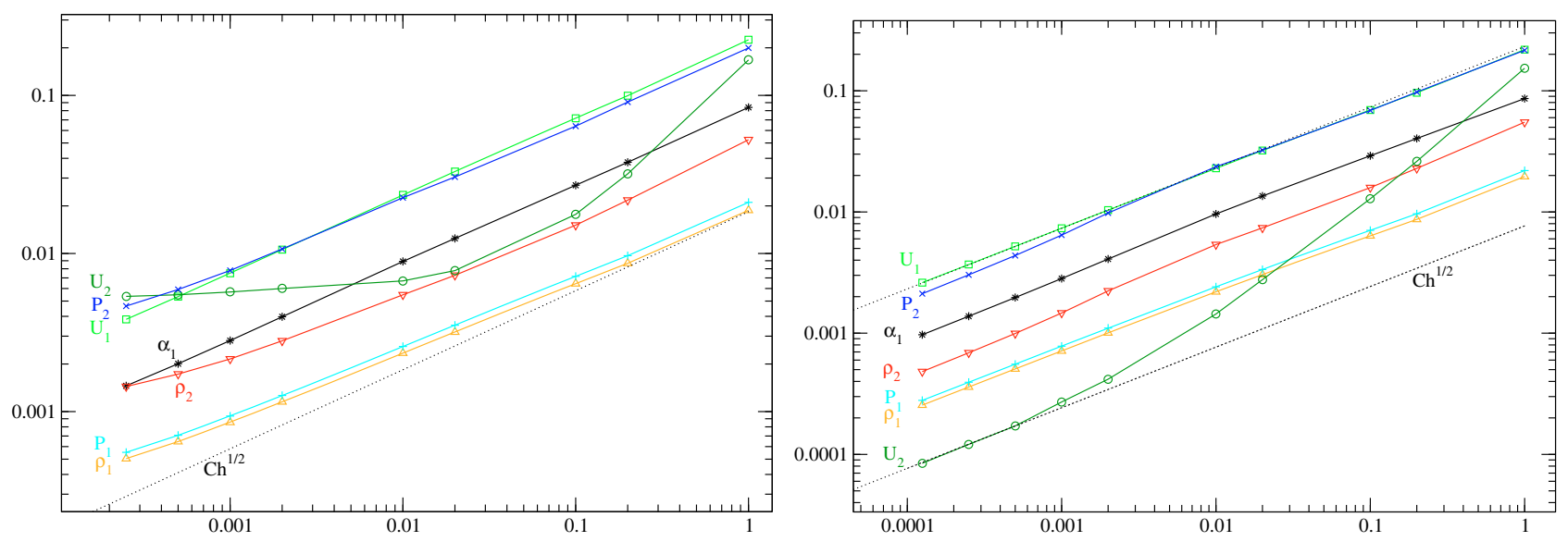

Figure 9. Test 4: $L_{1}$ norm of the error for scheme $M R$ (left) and $W B R$ (right) schemes.

\section{Results with WBR scheme}

Using exactly the same meshes and a finer mesh with $8 \times 10^{5}$ cells, it occurs in Figure 9 (on the right side) that convergence towards the correct solution is now recovered with $W B R$. Moreover, we retrieve expected rates of convergence $\beta(\phi)$ that are close to $1 / 2$, since all components $\phi$ vary through at least one contact discontinuity in this fourth test case. 

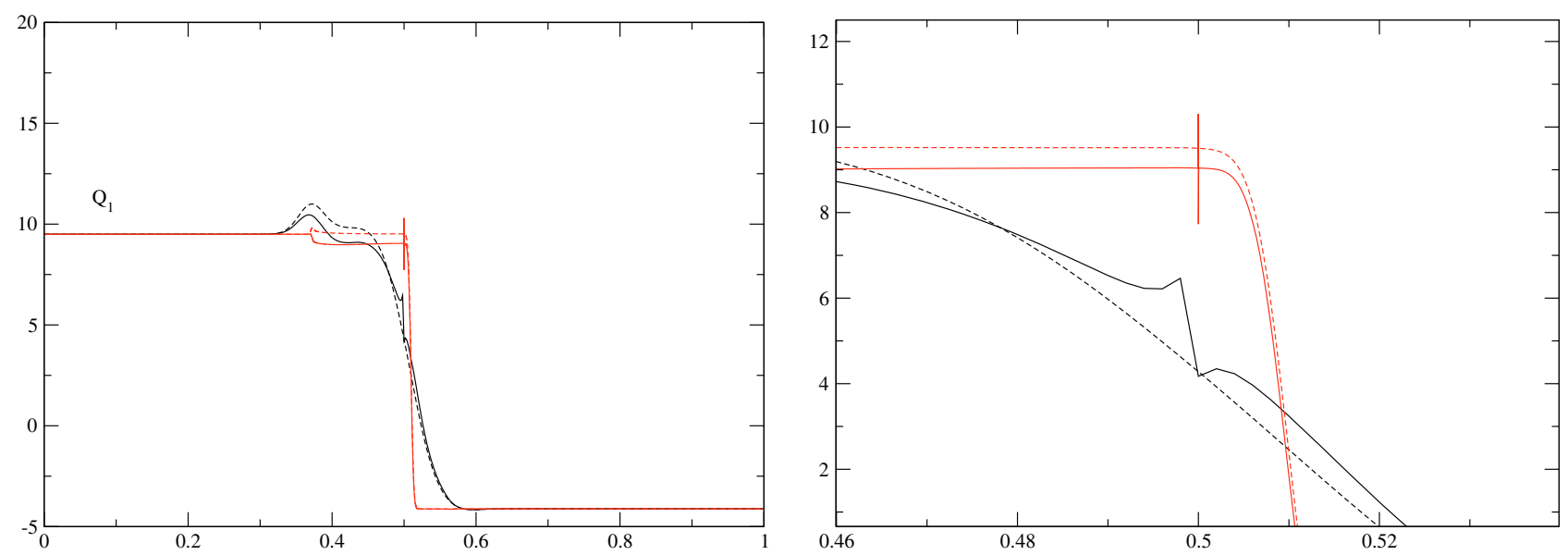

Figure 10. Mean momentum $\epsilon m_{1} U_{1}$ obtained with $M R$ and $W B R$ - dotted line - schemes. The coarse and fine meshes respectively contain 500 - in black - and 50000 - in red - cells. A zoom on $[0.46,0.54]$ on the right figure provides some more details.

\begin{tabular}{|c|c|c|c|c|}
\hline & $5 \times 10^{4}$ to $10^{5}$ cells & $10^{5}$ to $2 \times 10^{5}$ cells & $2 \times 10^{5}$ to $4 \times 10^{5}$ cells & $4 \times 10^{5}$ to $8 \times 10^{5}$ cells \\
\hline$\alpha_{1}$ & 0.535 & 0.521 & 0.512 & 0.506 \\
\hline$\rho_{1}$ & 0.495 & 0.493 & 0.495 & 0.496 \\
\hline$U_{1}$ & 0.499 & 0.495 & 0.497 & 0.497 \\
\hline$P_{1}$ & 0.496 & 0.494 & 0.496 & 0.496 \\
\hline$\rho_{2}$ & 0.607 & 0.562 & 0.529 & 0.516 \\
\hline$U_{2}$ & 0.625 & 0.655 & 0.505 & 0.521 \\
\hline$P_{2}$ & 0.607 & 0.560 & 0.529 & 0.516 \\
\hline
\end{tabular}

Local behaviour around the steady interface

We show in Figure 10 the behaviour of the discharge $\epsilon m_{1} U_{1}$ around the steady interface $x=0.5$ when computing the Riemann problem discussed above with $M R$ and $W B R$ schemes, while restricting to a coarse and a rather fine mesh. The $W B R$ scheme provides a reasonable local behaviour around the steady interface, and the amplitude of the reflected wave is indeed much smaller than the one occurring with the $M R$ scheme. Similar remarks hold for $\epsilon m_{2} U_{2}$ and other 0-Riemann invariants.

\section{INTERACTION OF FLUID WAVES WITH THE STEADY INTERFACE}

We now examine two tests where waves issuing from the free medium propagate towards the porous interface and interact with it. Though we have no analytic solution available in that case, it represents some relevant pattern for industrial applications. The validation of approximations provided by schemes will be evaluated by focusing on the Riemann invariants of the steady wave computed within each cell, when the flow is almost steady around the interface. Once again, the CFL number is equal to $1 / 2$ in all cases.

\section{Fifth test case}

This test case corresponds to a rough representation of a loss of coolant accident, where we focus on the propagation of the rarefaction wave that will hit a free/porous interface separating the pipe from the steam generator. The computational domain includes a free region $(\epsilon(x<0.35)=1)$ on the left side of an interface located at $x=0.35$, and a porous region $(\epsilon(x>0.35)=0.6)$ on the right side of the latter interface. The whole computational domain thus corresponds to $x \in[0 ; 1]$. The pipe is suddenly broken around $x=0.30$, 

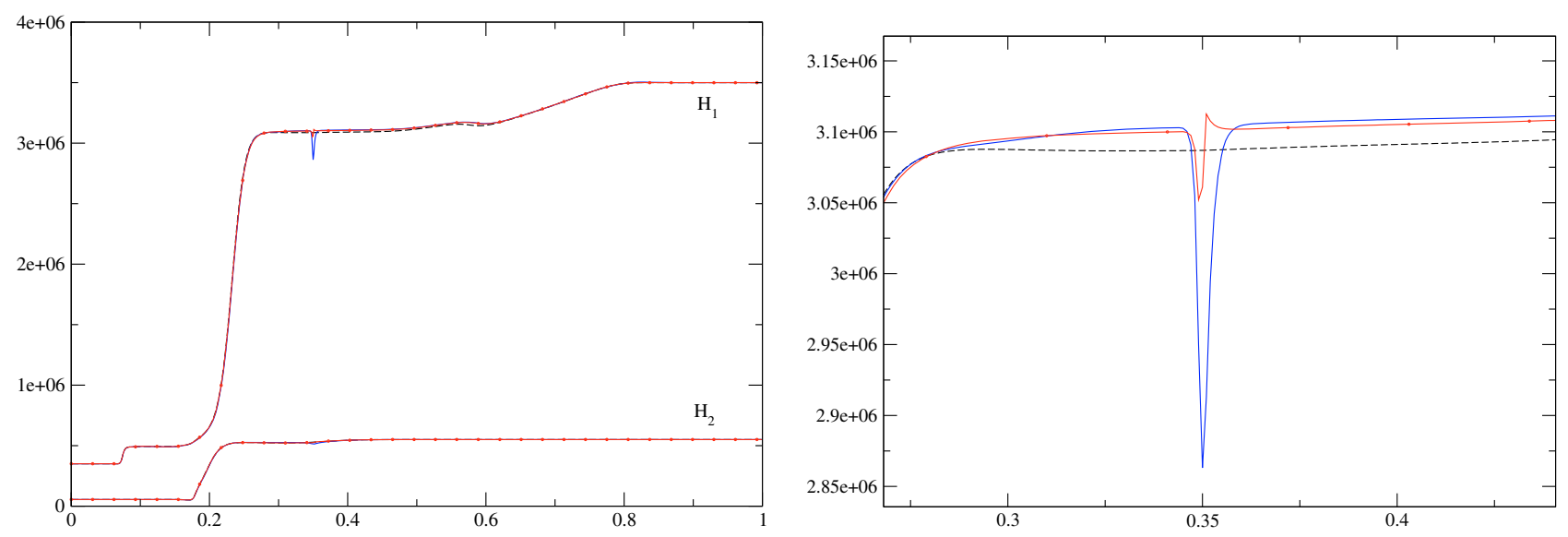

FiguRE 11. Test case 5: Riemann invariants $H_{1}$ and $H_{2}$, using a mesh with 1000 cells. The blue line, the red line with circles and the dotted black line correspond to $R, M R$ and $W B R$ schemes respectively. A zoom around the steady interface is displayed on the right for $H_{1}$.

at the beginning of the computation $(t=0)$. Denoting by $L, R$ the left and right states on both sides of the interface $x=0.30$, the initial conditions are:

\begin{tabular}{|c|c|c|}
\hline & Left state $L$ & Right state $R$ \\
\hline$\epsilon$ & 1 & 0.6 \\
\hline$\alpha_{1}$ & 0.95 & 0.05 \\
\hline$\rho_{1}$ & \multicolumn{2}{|c|}{1} \\
\hline$U_{1}$ & \multicolumn{2}{|c|}{0} \\
\hline$P_{1}$ & $1 \times 10^{5}$ & $1 \times 10^{6}$ \\
\hline$\rho_{2}$ & \multicolumn{2}{|c|}{0} \\
\hline$U_{2}$ & \multicolumn{2}{|c|}{$1 \times 10^{6}$} \\
\hline$P_{2}$ & $1 \times 10^{5}$ & 1 \\
\hline
\end{tabular}

Figures 11 and 12 show the behaviour of the Riemann invariants $H_{1}, H_{2}, s_{1}$ and $s_{2}$, when the rarefaction has passed the free/porous interface. The mesh contains 1000 regular cells. Subscript 2 now refers to the water phase. The best results are once again obtained with the $W B R$ scheme. Those pertaining to the $M R$ scheme are less spurious than those corresponding to the Rusanov scheme, but show again a non-monotone behaviour.

\section{Sixth test case}

The initial conditions for this last test case are given in the table below. The computational domain corresponds to $x \in[0 ; 1]$. The interface between codes is situated at $x=0.67$, and the porous medium is still on the right side of this interface. Both phases are at rest at the beginning of the computation, and $L, R$ states denote the initial states on the left and right side of $x=0.65$ respectively. Results for $H_{1}, H_{2}, s_{1}$ and $s_{2}$ are displayed in Figures 13 and 14. These have been plotted after the right-going shock waves and the right-going void fraction contact discontinuity have passed the coupling interface $(x=0.67)$. 

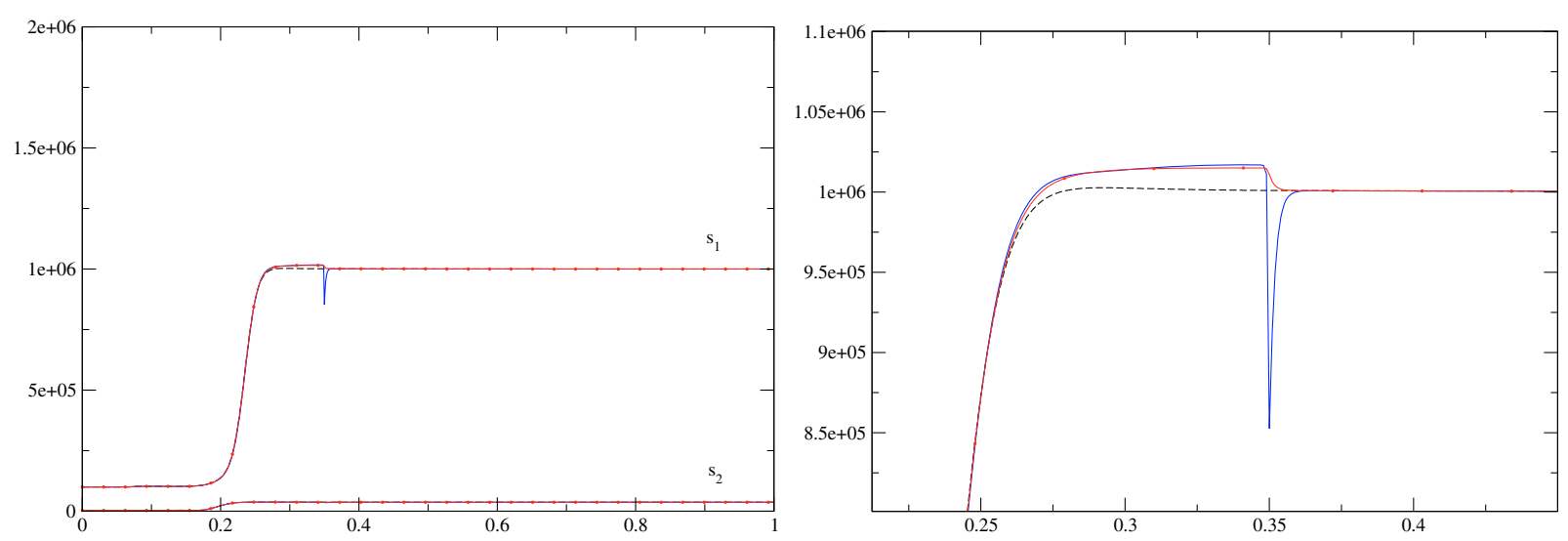

Figure 12. Test case 5: Riemann invariants $s_{1}$ and $s_{2}$, using a mesh with 1000 cells. The blue line, the red line with circles and the dotted black line correspond to $R, M R$ and $W B R$ schemes respectively. A zoom around the steady interface is displayed on the right for $s_{1}$.
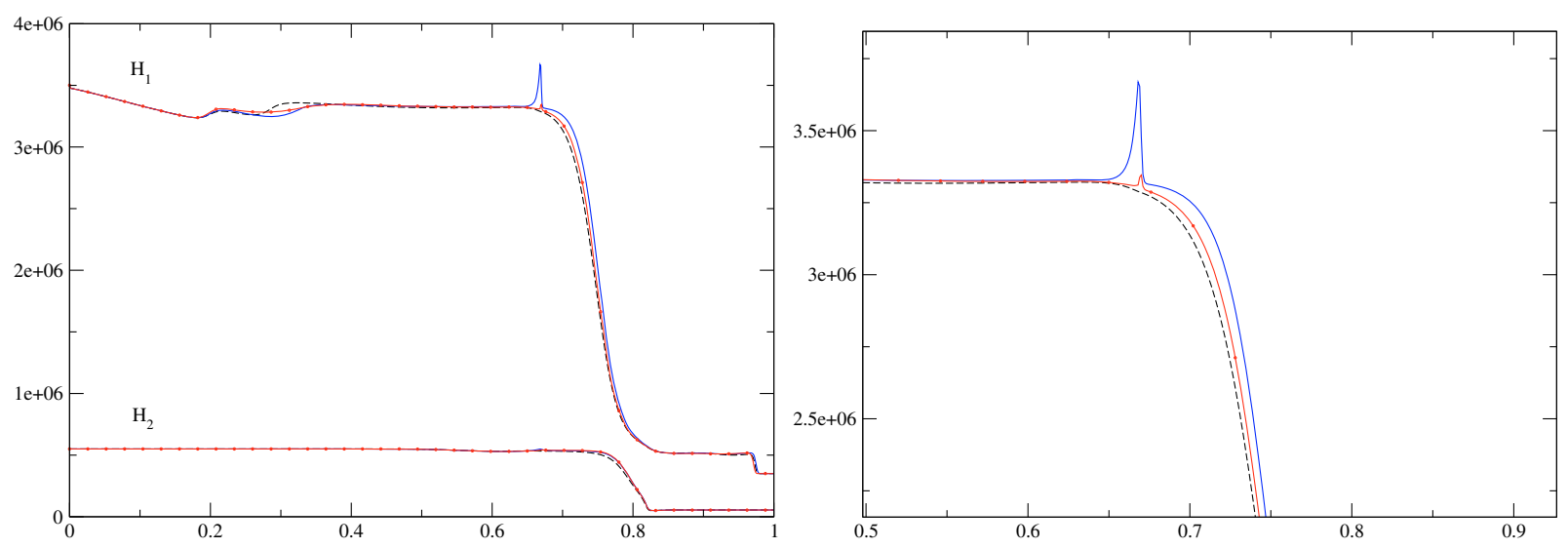

Figure 13. Test case 6: Riemann invariants $H_{1}$ and $H_{2}$, using a mesh with 1000 cells. The blue line, the red line with circles and the dotted black line correspond to $R, M R$ and $W B R$ schemes respectively. A zoom around the steady interface is displayed on the right for $H_{1}$.

\begin{tabular}{|c|c|c|}
\hline & Left state $L$ & Right state $R$ \\
\hline$\epsilon$ & 1 & 0.6 \\
\hline$\alpha_{1}$ & 0.05 & 0.95 \\
\hline$\rho_{1}$ & \multicolumn{2}{|c|}{1} \\
\hline$U_{1}$ & \multicolumn{2}{|c|}{0} \\
\hline$P_{1}$ & $1 \times 10^{6}$ & $1 \times 10^{5}$ \\
\hline$\rho_{2}$ & \multicolumn{2}{|c|}{0} \\
\hline$U_{2}$ & \multicolumn{2}{|c|}{0} \\
\hline$P_{2}$ & $1 \times 10^{6}$ & $1 \times 10^{5}$ \\
\hline
\end{tabular}

This last test case confirms that the behaviour of $W B R$ scheme is indeed fair. 

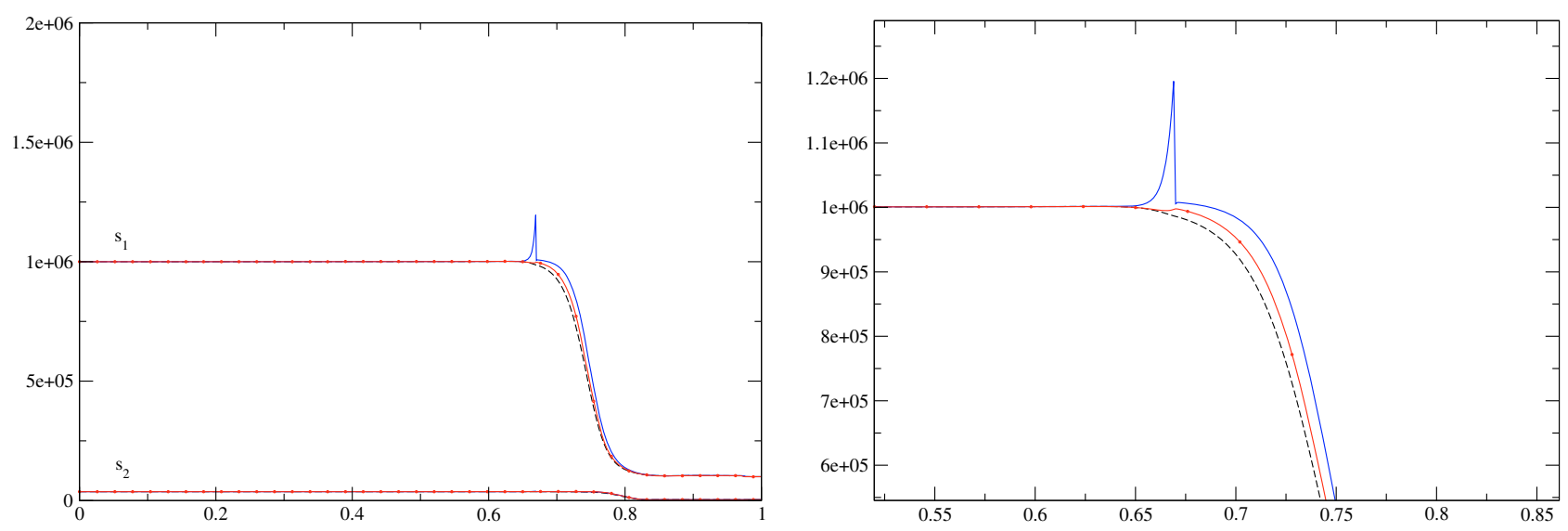

Figure 14. Test case 6: Riemann invariants $s_{1}$ and $s_{2}$, using a mesh with 1000 cells. The blue line, the red line with circles and the dotted black line correspond to $R, M R$ and $W B R$ schemes respectively. A zoom around the steady interface is displayed on the right for $s_{1}$.

\section{Conclusion}

- None among the first two schemes $R$ and $M R$ converges towards the correct solution when refining the mesh, if one computes approximations of solutions in very simple one-dimensional Riemann problems involving distinct values of porosity, such as those introduced in Section 3. Actually, the continuity that is enforced by the Rusanov scheme seems to inhibit the correct convergence when focusing on models for porous media. This is not due to the complexity inherent to the two-fluid approach, and the poor behaviour of these schemes in the framework of Euler equations in variable cross section flows has already been pointed out in the literature (see [28] for instance). However, and to the knowledge of authors, it had never been clearly stated that this drawback was not only annoying for coarse meshes, but also when tackling very fine meshes.

- On the contrary, the $W B R$ scheme, which inherits the spirit of well-balanced schemes combined with the inner stability of Rusanov-like fluxes, provides an extremely simple and useful tool for such computations in porous media. The expected rates of convergence are retrieved, focusing on so-called first-order schemes, and the nice behaviour of the discrete cell-values of Riemann invariants of the standing wave around the free/porous interface renders the scheme quite appealing. We recall that this scheme is inspired by the scheme introduced in [28] for Euler equations in variable cross section ducts (see [29] too). It also means that Property 6 is far from being sufficient, and that the dynamical well-balanced Property 7 enjoyed by $W B R$, the continuous counterpart of which is Property 3 , seems mandatory to obtain convergence towards the correct solution.

A straightforward consequence for the NEPTUNE project is that at least one meaningful scheme is available in order to perform the interfacial unsteady coupling of codes that aim at providing approximations of PDEs in free and porous medium respectively.

Among possible improvements and current work in progress in this area, we would like to mention that:

- In order to achieve a better coupling, and more precisely a more relevant treatment of the free/porous interface, we need to provide more physical coupling conditions for momentum equations that might account for the head loss of momentum. This is known to be very tricky, and it is not clear whether direct simulations will in fine provide useful tools in practice. A similar remark holds for heat losses through the free/porous interface.

- We also would like to emphasize that an approximate Godunov scheme that inherits a similar wellbalanced property may be constructed. This one also makes use of basic ideas introduced by Greenberg 
and Leroux [21], while substituting the approximate Godunov VFRoe-ncv fluxes introduced in [7] to the exact Godunov "fluxes" through the cell interfaces, instead of Rusanov fluxes used in the present work. This of course requires using a suitable variable, as achieved for instance in $[13,24]$ for shallow water equations with topography and isentropic Euler equations in a porous medium. The main advantage of the latter solver is that its accuracy is increased when compared with the scheme introduced in [28]. Its extension to the framework of two-phase two-fluid models is currently in progress and seems feasible, and its counterpart for homogeneous flows also seems rather satisfactory [23].

- The extension to the framework of three phase flows in a porous medium is also feasible, following [25] for instance.

- The extension of the present WBR scheme to the framework of three-dimensional flows is straightforward (see [15]), and it provides satisfactory results, while preserving the basic positivity results given in Property 8.

\section{A. Construction of solutions of the Riemann problem}

We detail here the construction of the solutions used in the main part of the paper. We start with a given state $L$, and then successively calculate states $A, B$ and $R$. We assume here that the EOS for each phase is given by:

$$
\left(\gamma_{k}-1\right) \rho_{k} e_{k}=P_{k} \text {. }
$$

\section{A.1. Given parameters}

We assume that the left state $\left(\epsilon_{L}, \alpha_{2_{L}}, \rho_{1_{L}}, \rho_{2_{L}}, P_{1_{L}}, P_{2_{L}}, U_{1_{L}}, U_{2_{L}}\right)$ is given (with $\left(U_{2}\right)_{L}>0$ ), and we also choose $\epsilon_{R}$ and $\alpha_{2_{R}}$, so that: $\epsilon_{L} \epsilon_{R} \neq 0, \alpha_{2_{L}} \alpha_{2_{R}} \neq 0$ and $\alpha_{1_{L}} \alpha_{1_{R}} \neq 0$.

\section{A.2. Construction of the first intermediate state $A$}

We first obviously get:

$$
\epsilon_{A}=\epsilon_{R} .
$$

Since states $L$ and $A$ are separated by the steady LD wave associated with $\lambda_{0}=0$, their connection is thus ensured by Riemann invariants $I_{n}^{0}(W), n=1,2, \ldots, 7$.

- Using Riemann invariant $I_{1}^{0}(W)$ provides:

$$
\alpha_{2_{A}}=\alpha_{2_{L}}
$$

due to the fact that $U_{2_{A}}>0$ (see below).

- Riemann invariants $I_{3}^{0}(W)$ and $I_{6}^{0}(W)$ enable to write:

$$
U_{1_{A}}=\frac{\left(\epsilon_{L}\right)\left(\rho_{1_{L}}\right)\left(U_{1_{L}}\right)}{\left(\epsilon_{A}\right)\left(\rho_{1_{A}}\right)} ; \quad U_{2_{A}}=\frac{\left(\epsilon_{L}\right)\left(\rho_{2_{L}}\right)\left(U_{2_{L}}\right)}{\left(\epsilon_{A}\right)\left(\rho_{2_{A}}\right)} .
$$

Moreover, using Riemann invariants $I_{2}^{0}(W)$ and $I_{5}^{0}(W)$, we can express both pressures $P_{1_{A}}$ and $P_{2_{A}}$ in terms of $\rho_{1_{A}}$ and $\rho_{2_{A}}$ respectively:

$$
P_{1_{A}}=P_{1_{L}}\left(\frac{\rho_{1_{L}}}{\rho_{1_{A}}}\right)^{-\gamma_{1}} ; \quad P_{2_{A}}=P_{2_{L}}\left(\frac{\rho_{2_{L}}}{\rho_{2_{A}}}\right)^{-\gamma_{2}} .
$$

Hence, substituting all these expressions in Riemann invariants $I_{4}^{0}(W)$ and $I_{7}^{0}(W)$, we need to find solutions of $\left(g_{A}\right)_{k}(X)=0$ where:

$$
\left(g_{A}\right)_{k}(X)=\frac{\gamma_{k}}{\gamma_{k}-1} s_{k_{L}}\left(X^{\gamma_{k}-1}-\rho_{k_{L}}^{\gamma_{k}-1}\right)+\frac{1}{2}\left(\left(\frac{\epsilon_{L}}{\epsilon_{R}}\right)^{2}\left(\frac{\rho_{k_{L}}}{X}\right)^{2}-1\right) U_{k_{L}}^{2}
$$

wrt to $\rho_{k_{A}}$. 
If $U_{k_{L}}=0,\left(g_{A}\right)_{k}(X)=0$ admits a unique solution: $\rho_{k_{A}}=\rho_{k_{L}}$. Otherwise, the function $\left(g_{A}\right)_{k}(X)$ is decreasing in $\left.] 0, X_{\min _{A}, k}\right]$, and then increasing in $] X_{\min _{A}, k},+\infty[$, setting:

$$
X_{\min _{A}, k}=\left(\frac{U_{k_{L}}^{2}\left(\frac{\epsilon_{L}}{\epsilon_{R}}\right)^{2}\left(\rho_{k}\right)_{L}^{2}}{\gamma_{k}\left(s_{k}\right)_{L}}\right)^{\frac{1}{\gamma_{k}+1}}
$$

and we also have $\lim \left(g_{A}\right)_{k}(X)=+\infty$ when $X$ tends to 0 (or $+\infty$ ). Thus, $\left(g_{A}\right)_{k}(X)=0$ may admit two solutions in the general case, no solution if $\left(g_{A}\right)_{k}\left(X_{\min _{A}, k}\right)>0$.

Once $\rho_{k_{A}}$ has been calculated, we may use initial expressions to deduce $U_{k_{A}}$ and $P_{k_{A}}$, for $k=1,2$.

In practice, for the test cases defined in the main section, we have used the solution $\rho_{k_{A}}$ that has the same sonic regime than $\rho_{k_{L}}$.

\section{A.3. Construction of the intermediate state $B$}

States $A$ and $B$ are separated by the contact discontinuity associated with $\lambda_{1}=V_{I}=U_{2}$. The jump of $\alpha_{2}$ is located here, so that: $\alpha_{2_{B}}=\alpha_{2_{R}}$.

The remaining components of the intermediate state $B$ are calculated through Riemann invariants $I_{n}^{1}(W)$, $n=1,2, \ldots, 6$.

- First of all, by virtue of $I_{1}^{1}(W)$, we obviously have $\epsilon_{B}=\epsilon_{A}=\epsilon_{R}$.

Owing to $I_{3}^{1}(W)$, we know that $U_{2_{B}}=U_{2_{A}}$. Moreover, $I_{4}^{1}(W)$ provides $U_{1_{B}}$ in terms of $\rho_{1_{B}}$ :

$$
U_{1_{B}}=\frac{m_{1_{A}}}{m_{1_{B}}}\left(U_{1_{A}}-U_{2_{A}}\right)+U_{2_{A}}
$$

and $I_{2}^{1}(W)$ enforces: $P_{1_{B}}=P_{1_{A}}\left(\frac{\rho_{1_{A}}}{\rho_{1_{B}}}\right)^{-\gamma_{1}}$.

Eventually, the expression $I_{5}^{1}(W)$ enables to calculate $P_{2_{B}}$ as:

$$
P_{2_{B}}=\frac{1}{\alpha_{2_{R}}}\left(\alpha_{1_{L}} P_{1_{A}}+\alpha_{2_{L}} P_{2_{A}}+m_{1_{A}} U_{1_{A}}\left(U_{1_{A}}-U_{2_{A}}\right)-\alpha_{1_{B}} P_{1_{B}}-m_{1_{B}} U_{1_{B}}\left(U_{1_{B}}-U_{2_{B}}\right)\right) .
$$

Hence, substituting all these expressions in the last Riemann invariant $I_{6}^{1}(W)$, we conclude that $\rho_{1_{B}}$ must be a solution of $g_{B}(X)=0$ where:

$$
g_{B}(X)=\frac{\gamma_{1}}{\gamma_{1}-1} s_{1_{A}}\left(X^{\gamma_{1}-1}-\rho_{1_{A}}^{\gamma_{1}-1}\right)+\frac{1}{2}\left(\left(\frac{\alpha_{1_{L}}}{\alpha_{1_{R}}}\right)^{2}\left(\frac{\rho_{1_{A}}}{X}\right)^{2}-1\right)\left(U_{1_{A}}-U_{2_{A}}\right)^{2} .
$$

- Once again, if $U_{1_{A}}=U_{2_{A}}$, there exists only one solution $\rho_{1_{B}}=\rho_{1_{A}}$.

- Otherwise, we need to solve $g_{B}(X)=0$. The procedure is exactly the same as the one discussed in the previous section, where we solve $g_{A}(X)=0$ to calculate state $A$.

- Obviously, owing to the structure of the LD field associated with $\lambda_{1}=V_{I}=U_{2}$, we need to impose the value of $\rho_{2_{B}}$. Thus, state $B$ is completely determined. 


\section{A.4. Construction of the right state $R$}

We have assumed that states $B$ and $R$ are separated by a GNL wave associated with $\lambda_{7}=U_{2}+c_{2}$. We also recall that $\epsilon_{R}$ and $\alpha_{R}$ have already been set.

This wave is a "ghost" wave for variables in phase 1. Thus:

- $\rho_{1_{R}}=\rho_{1_{B}} ; \quad U_{1_{R}}=U_{1_{B}} ; \quad P_{1_{R}}=P_{1_{B}}$.

Turning then to components in phase 2 , we face a single-phase problem. Thus we get:

- $\rho_{2_{R}}=\frac{1}{z_{R}} \rho_{2_{B}}$ where $z_{R} \geqslant 1$ must be set, so that $\lambda_{7}=U_{2}+c_{2}$ is a shock wave.

- $U_{2_{R}}=U_{2_{B}}-c_{2_{R}}\left(\frac{\left(\beta_{2}+1\right)\left(z_{R}-1\right)^{2}}{\gamma_{2} z_{R}\left(\beta_{2}-z_{R}\right)}\right)^{\frac{1}{2}}$ where $\beta_{2}=\frac{\gamma_{2}+1}{\gamma_{2}-1}$ and $c_{2_{R}}=\left(\frac{\gamma_{2} P_{2_{R}}}{\rho_{2_{R}}}\right)^{\frac{1}{2}}$

- $P_{2_{R}}=P_{2_{B}} \frac{\beta_{2}-z_{R}}{\beta_{2} z_{R}-1}$.

\section{B. MAIN PROPERTIES OF SCHEMES $R, M R$ AND $W B R$}

This appendix is devoted to the proofs of main properties of Section 5.

\section{B.1. Property 5}

In a free medium, schemes $R, M R$ and $W B R$ are identical. Thus, if $\epsilon_{i}=\epsilon_{0}$ for all $i$, we check it for scheme $R$. Assume that for given $n,\left(U_{k}\right)_{i}^{n}=U_{0}$ and $\left(P_{k}\right)_{i}^{n}=P_{0}$ for all $i(k=1,2)$. We prove now that $\left(U_{1}\right)_{i}^{n+1}=\left(U_{2}\right)_{i}^{n+1}=U_{0}$ and also: $\left(P_{1}\right)_{i}^{n+1}=\left(P_{2}\right)_{i}^{n+1}=P_{0}$, if $\epsilon_{i}=\epsilon_{0}$.

The discrete equations that update $\left(\alpha_{k}\right)_{i}^{n+1},\left(m_{k}\right)_{i}^{n+1},\left(m_{k} U_{k}\right)_{i}^{n+1}$ are:

$$
\begin{aligned}
h_{i}\left(\left(\alpha_{k}\right)_{i}^{n+1}-\left(\alpha_{k}\right)_{i}^{n}\right)+ & \Delta t^{n} U_{0}\left(\left(\overline{\alpha_{k}}\right)_{i+1 / 2}^{n}-\left(\overline{\alpha_{k}}\right)_{i-1 / 2}^{n}\right) \\
& -\frac{1}{2} \Delta t^{n}\left(r_{i+1 / 2}^{n}\left(\left(\alpha_{k}\right)_{i+1}^{n}-\left(\alpha_{k}\right)_{i}^{n}\right)-r_{i-1 / 2}^{n}\left(\left(\alpha_{k}\right)_{i}^{n}-\left(\alpha_{k}\right)_{i-1}^{n}\right)\right)=0 \\
h_{i} \epsilon_{0}\left(\left(m_{k}\right)_{i}^{n+1}-\left(m_{k}\right)_{i}^{n}\right)+ & \Delta t^{n} \epsilon_{0} U_{0}\left(\left(\overline{m_{k}}\right)_{i+1 / 2}^{n}-\left(\overline{m_{k}}\right)_{i-1 / 2}^{n}\right) \\
- & \frac{1}{2} \Delta t^{n} \epsilon_{0}\left(r_{i+1 / 2}^{n}\left(\left(m_{k}\right)_{i+1}^{n}-\left(m_{k}\right)_{i}^{n}\right)-r_{i-1 / 2}^{n}\left(\left(m_{k}\right)_{i}^{n}-\left(m_{k}\right)_{i-1}^{n}\right)\right)=0 \\
h_{i} \epsilon_{0}\left(\left(m_{k} U_{k}\right)_{i}^{n+1}-\left(m_{k} U_{k}\right)_{i}^{n}\right)+ & \Delta t^{n} \epsilon_{0}\left(U_{0}\right)^{2}\left(\left(\overline{m_{k}}\right)_{i+1 / 2}^{n}-\left(\overline{m_{k}}\right)_{i-1 / 2}^{n}\right) \\
& -\frac{1}{2} \Delta t^{n} \epsilon_{0} U_{0}\left(r_{i+1 / 2}^{n}\left(\left(m_{k}\right)_{i+1}^{n}-\left(m_{k}\right)_{i}^{n}\right)-r_{i-1 / 2}^{n}\left(\left(m_{k}\right)_{i}^{n}-\left(m_{k}\right)_{i-1}^{n}\right)\right)=0 .
\end{aligned}
$$

Multiplying (B.2) by $U_{0}$, and then subtracting the result to the discrete equation of energy in phase $k$, and using $\left(U_{k}\right)_{i}^{n}=U_{0}$, we obtain:

which guarantees that for all $i$ :

$$
\left(m_{k}\right)_{i}^{n+1}\left(\left(U_{k}\right)_{i}^{n+1}-U_{0}\right)=0
$$

$$
\left(U_{k}\right)_{i}^{n+1}=U_{0} .
$$

Using now (B.4), multiplying (B.3) by a factor $\frac{U_{0}}{2}$, and subtracting the resulting equation to (B.3) leads to:

$$
\begin{aligned}
h_{i} \epsilon_{0}\left(\left(m_{k} e_{k}\right)_{i}^{n+1}-\left(m_{k} e_{k}\right)_{i}^{n}\right)+ & \Delta t^{n} \epsilon_{0} U_{0}\left(\left(\overline{m_{k} e_{k}}\right)_{i+1 / 2}^{n}-\left(\overline{m_{k} e_{k}}\right)_{i-1 / 2}^{n}\right) \\
& -\frac{1}{2} \Delta t^{n} \epsilon_{0}\left(r_{i+1 / 2}^{n}\left(\left(m_{k} e_{k}\right)_{i+1}^{n}-\left(m_{k} e_{k}\right)_{i}^{n}\right)-r_{i-1 / 2}^{n}\left(\left(m_{k} e_{k}\right)_{i}^{n}-\left(m_{k} e_{k}\right)_{i-1}^{n}\right)\right)=0 .
\end{aligned}
$$

We now need to introduce the EOS that takes the form: $\rho_{k} e_{k}\left(P_{k}, \rho_{k}\right)=a_{k, 0} \rho_{k}+g_{k}\left(P_{k}\right)$ in each phase $k$. 
Since $\left(P_{k}\right)_{i}^{n}=P_{0}$ for all $i$, this implies $\left(g_{k}\left(P_{k}\right)\right)_{i}^{n}=g_{k}\left(P_{0}\right)$. Thus, replacing $m_{k} e_{k}$ by $a_{k, 0} m_{k}+\alpha_{k} g_{k}\left(P_{k}\right)$ in (B.5), subtracting $a_{k, 0}$ times (B.2) and also $\epsilon_{0} g_{k}\left(P_{0}\right)$ times (B.1) to the discrete equation of energy (B.5), we get:

$$
\left(\alpha_{k}\right)_{i}^{n+1}\left(\left(g_{k}\left(P_{k}\right)\right)_{i}^{n+1}-g_{k}\left(P_{0}\right)\right)=0
$$

which clearly implies that for all $i$ :

$$
\left(P_{k}\right)_{i}^{n+1}=P_{0}
$$

This completes the proof of Property 5, which means that the three schemes $R, M R$ et $W B R$ preserve the discrete form of solution $S_{1}$ on any mesh.

\section{B.2. Property 6}

B.2.1. Scheme $M R$ preserves the discrete form of the basic solution $S_{2}$ on any mesh

We assume now that $\left(U_{1}\right)_{i}^{n}=\left(U_{2}\right)_{i}^{n}=0$ and $\left(P_{1}\right)_{i}^{n}=\left(P_{2}\right)_{i}^{n}=P_{0}$. We successively prove that $\left(U_{1}\right)_{i}^{n+1}=$ $\left(U_{2}\right)_{i}^{n+1}=0$ and $\left(P_{1}\right)_{i}^{n+1}=\left(P_{2}\right)_{i}^{n+1}=P_{0}$, whatever $\epsilon_{i}$ is.

Using initial data, the discrete forms of the void fraction/partial mass/momentum take the form:

$$
\begin{aligned}
& h_{i}\left(\left(\alpha_{k}\right)_{i}^{n+1}-\left(\alpha_{k}\right)_{i}^{n}\right)-\frac{1}{2} \Delta t^{n}\left(r_{i+1 / 2}^{n} \frac{\hat{\epsilon}_{i+1 / 2}}{\epsilon_{i}}\left(\left(\alpha_{k}\right)_{i+1}^{n}-\left(\alpha_{k}\right)_{i}^{n}\right)-r_{i-1 / 2}^{n} \frac{\hat{\epsilon}_{i-1 / 2}}{\epsilon_{i}}\left(\left(\alpha_{k}\right)_{i}^{n}-\left(\alpha_{k}\right)_{i-1}^{n}\right)\right)=0 \\
& h_{i} \epsilon_{i}\left(\left(m_{k}\right)_{i}^{n+1}-\left(m_{k}\right)_{i}^{n}\right)-\frac{1}{2} \Delta t^{n}\left(r_{i+1 / 2}^{n} \hat{\epsilon}_{i+1 / 2}\left(\left(m_{k}\right)_{i+1}^{n}-\left(m_{k}\right)_{i}^{n}\right)-r_{i-1 / 2}^{n} \hat{\epsilon}_{i-1 / 2}\left(\left(m_{k}\right)_{i}^{n}-\left(m_{k}\right)_{i-1}^{n}\right)\right)=0 .
\end{aligned}
$$

Owing to the fact that both $\left(P_{k}\right)_{i}^{n}=P_{0}$ and $\left(U_{k}\right)_{i}^{n}=0$, we also get:

$$
h_{i} \epsilon_{i}\left(\left(m_{k} U_{k}\right)_{i}^{n+1}-\left(m_{k} U_{k}\right)_{i}^{n}\right)=0
$$

and thus:

$$
\left(U_{k}\right)_{i}^{n+1}=0 .
$$

Still using the EOS such that: $\rho_{k} e_{k}\left(P_{k}, \rho_{k}\right)=a_{k, 0} \rho_{k}+g_{k}\left(P_{k}\right)$, we get: $m_{k} e_{k}=a_{k, 0} m_{k}+\alpha_{k} g_{k}\left(P_{k}\right)$ for $k=1,2$. Thus, multiplying (B.8) by $a_{k, 0}$, and subtracting the resulting equation to the energy equation, we get:

$$
\begin{aligned}
& h_{i} \epsilon_{i}\left(\left(\alpha_{k} g_{k}\left(P_{k}\right)\right)_{i}^{n+1}-g_{k}\left(P_{0}\right)\left(\alpha_{k}\right)_{i}^{n}\right) \\
& -\frac{1}{2} \Delta t^{n} g_{k}\left(P_{0}\right)\left(r_{i+1 / 2}^{n} \hat{\epsilon}_{i+1 / 2}\left(\left(\alpha_{k}\right)_{i+1}^{n}-\left(\alpha_{k}\right)_{i}^{n}\right)-r_{i-1 / 2}^{n} \hat{\epsilon}_{i-1 / 2}\left(\left(\alpha_{k}\right)_{i}^{n}-\left(\alpha_{k}\right)_{i-1}^{n}\right)\right)=0 .
\end{aligned}
$$

Hence, multiplying (B.7) by $\epsilon_{i} g_{k}\left(P_{0}\right)$ and subtracting the result to (B.11), we get:

$$
\left(\alpha_{k}\right)_{i}^{n+1}\left(\left(g_{k}\left(P_{k}\right)\right)_{i}^{n+1}-g_{k}\left(P_{0}\right)\right)=0
$$

which provides the expected result for all $i$ :

$$
\left(P_{k}\right)_{i}^{n+1}=P_{0}
$$

This completes the proof for scheme $M R$.

\section{B.2.2. Scheme $W B R$ preserves the discrete form of the basic solution $S_{2}$ on any mesh}

The scheme $W B R$ ensures that we have: $\left(\alpha_{2}\right)_{i+1 / 2,-}^{n}=\left(\alpha_{2}\right)_{i+1}^{n},\left(\alpha_{2}\right)_{i-1 / 2,+}^{n}=\left(\alpha_{2}\right)_{i-1}^{n}$. Moreover, due to the fact that $\left(U_{k}\right)_{i}^{n}=0$, we get: $\left(U_{k}\right)_{i+1 / 2,-}^{n}=\left(U_{k}\right)_{i-1 / 2,+}^{n}=0$, and thus: $\left(\rho_{k}\right)_{i+1 / 2,-}^{n}=\left(\rho_{k}\right)_{i+1}^{n},\left(\rho_{k}\right)_{i-1 / 2,+}^{n}=$ $\left(\rho_{k}\right)_{i-1}^{n}$, together with: $\left(s_{k}\right)_{i+1 / 2,-}^{n}=\left(s_{k}\right)_{i+1}^{n},\left(s_{k}\right)_{i-1 / 2,+}^{n}=\left(s_{k}\right)_{i-1}^{n}$. As a consequence, we get: $\left(P_{k}\right)_{i+1 / 2,-}^{n}=$ $\left(P_{k}\right)_{i+1}^{n}=P_{0}$, and $\left(P_{k}\right)_{i-1 / 2,+}^{n}=\left(P_{k}\right)_{i-1}^{n}=P_{0}$. Hence, the discrete equations for the void fraction, the partial 
masses and the momentum can be simplified, which yields:

$$
\begin{gathered}
h_{i}\left(\left(\alpha_{k}\right)_{i}^{n+1}-\left(\alpha_{k}\right)_{i}^{n}\right)-\frac{1}{2} \Delta t^{n}\left(\left(r_{W B}\right)_{i+1 / 2}^{n}\left(\left(\alpha_{k}\right)_{i+1}^{n}-\left(\alpha_{k}\right)_{i}^{n}\right)-\left(r_{W B}\right)_{i-1 / 2}^{n}\left(\left(\alpha_{k}\right)_{i}^{n}-\left(\alpha_{k}\right)_{i-1}^{n}\right)\right)=0 \\
h_{i}\left(\left(m_{k}\right)_{i}^{n+1}-\left(m_{k}\right)_{i}^{n}\right)-\frac{1}{2} \Delta t^{n}\left(\left(r_{W B}\right)_{i+1 / 2}^{n}\left(\left(m_{k}\right)_{i+1}^{n}-\left(m_{k}\right)_{i}^{n}\right)-\left(r_{W B}\right)_{i-1 / 2}^{n}\left(\left(m_{k}\right)_{i}^{n}-\left(m_{k}\right)_{i-1}^{n}\right)\right)=0 \\
h_{i}\left(\left(m_{k} U_{k}\right)_{i}^{n+1}-\left(m_{k} U_{k}\right)_{i}^{n}\right)=0
\end{gathered}
$$

and eventually:

$$
\left(U_{k}\right)_{i}^{n+1}=0 .
$$

We still use the EOS in agreement with: $\rho_{k} e_{k}\left(P_{k}, \rho_{k}\right)=a_{k, 0} \rho_{k}+g_{k}\left(P_{k}\right)$. Initial conditions are such that the discrete energy equation reduces to:

$$
\begin{aligned}
h_{i}\left(\left(m_{k} e_{k}\right)_{i}^{n+1}-\left(m_{k} e_{k}\right)_{i}^{n}\right)-\frac{1}{2} \Delta t^{n}\left(( r _ { W B } ) _ { i + 1 / 2 } ^ { n } \left(\left(m_{k} e_{k}\right)_{i+1}^{n}\right.\right. & \left.-\left(m_{k} e_{k}\right)_{i}^{n}\right) \\
& \left.-\left(r_{W B}\right)_{i-1 / 2}^{n}\left(\left(m_{k} e_{k}\right)_{i}^{n}-\left(m_{k} e_{k}\right)_{i-1}^{n}\right)\right)=0 .
\end{aligned}
$$

Once again, we multiply (B.14) by $a_{k, 0}$ (respectively (B.13) by $g_{k}\left(P_{0}\right)$ ), and subtract both to (B.17). We get:

$$
\left(\alpha_{k}\right)_{i}^{n+1}\left(\left(g_{k}\left(P_{k}\right)\right)_{i}^{n+1}-g_{k}\left(P_{0}\right)\right)=0
$$

which implies that for all $i$ :

$$
\left(P_{k}\right)_{i}^{n+1}=P_{0}
$$

\section{B.3. Property 7}

We assume that $\epsilon_{L} \neq \epsilon_{R}$, and also that the initial condition $\left(W_{L}, W_{R}\right)$ of the Riemann problem complies with:

The initial condition complies with:

$$
I_{k}^{0}\left(W_{L}\right)=I_{k}^{0}\left(W_{R}\right)
$$

$$
\begin{cases}W_{i}^{n}=W_{L} & \text { pour } \quad i \leq j \\ W_{i}^{n}=W_{R} & \text { pour } \quad i>j\end{cases}
$$

We wish to prove that $W_{i}^{n+1}=W_{i}^{n}$ whatever $i$ is. We only focus on the interface separating cells $j$ and $j+1$.

The assumption (B.18) implies that:

$$
\left(\alpha_{k}\right)_{j}^{n}=\left(\alpha_{k}\right)_{j+1}^{n}
$$

and this is of course true for other cells so that $\left(\alpha_{k}\right)_{i-1}^{n}=\left(\alpha_{k}\right)_{i}^{n}=\left(\alpha_{k}\right)_{i+1}^{n}$. As a consequence, we have $\left(\alpha_{2}\right)_{j+1 / 2,-}^{n}=\left(\alpha_{2}\right)_{j+1}^{n}=\left(\alpha_{2}\right)_{j}^{n}$, and $\left(\alpha_{2}\right)_{j-1 / 2,+}^{n}=\left(\alpha_{2}\right)_{j-1}^{n}=\left(\alpha_{2}\right)_{j}^{n}$. The discrete equation for the void fraction thus yields:

$$
\left(\alpha_{k}\right)_{i}^{n+1}=\left(\alpha_{k}\right)_{i}^{n} \quad \forall i .
$$

The $W B R$ scheme also enforces that:

$$
\left(\epsilon \alpha_{k} \rho_{k} U_{k}\right)_{j+1 / 2,-}^{n}=\left(\epsilon \alpha_{k} \rho_{k} U_{k}\right)_{j+1}^{n}
$$

and thus:

$$
(\epsilon)_{j}\left(\alpha_{k} \rho_{k} U_{k}\right)_{j+1 / 2,-}^{n}=(\epsilon)_{j+1}\left(\alpha_{k} \rho_{k} U_{k}\right)_{j+1}^{n} .
$$


The assumption (B.18) enables to state that:

$$
\epsilon_{j+1}\left(\alpha_{k} \rho_{k} U_{k}\right)_{j+1}^{n}=\epsilon_{j}\left(\alpha_{k} \rho_{k} U_{k}\right)_{j}^{n}
$$

Relations (B.23), (B.24) and (B.21) lead to:

$$
\left(\rho_{k}\right)_{j+1 / 2,-}^{n}\left(U_{k}\right)_{j+1 / 2,-}^{n}=\left(\rho_{k}\right)_{j}^{n}\left(U_{k}\right)_{j}^{n}
$$

A similar work with Riemann invariants $s_{k}$ and $h_{k}$ gives:

$$
\begin{gathered}
\left(s_{k}\right)_{j+1 / 2,-}^{n}=\left(s_{k}\right)_{j+1}^{n}=\left(s_{k}\right)_{j}^{n} \\
h_{k}\left(\left(\rho_{k}\right)_{j+1 / 2,-}^{n},\left(U_{k}\right)_{j+1 / 2,-}^{n},\left(s_{k}\right)_{j+1 / 2,-}^{n}\right)=h_{k}\left(\left(\rho_{k}\right)_{j+1}^{n},\left(U_{k}\right)_{j+1}^{n},\left(s_{k}\right)_{j+1}^{n}\right)=h_{k}\left(\left(\rho_{k}\right)_{j}^{n},\left(U_{k}\right)_{j}^{n},\left(s_{k}\right)_{j}^{n}\right) .
\end{gathered}
$$

Since the scheme $W B R$ retains the solution which is in the same sonic regime, we may conclude, using constraints (B.26) and (B.27), that:

$$
\left\{\begin{array}{l}
\left(\rho_{k}\right)_{j+1 / 2,-}^{n}=\left(\rho_{k}\right)_{j}^{n} \\
\left(U_{k}\right)_{j+1 / 2,-}^{n}=\left(U_{k}\right)_{j}^{n} .
\end{array}\right.
$$

Using a similar process, we also have on the left side of cell $j$ :

$$
\left\{\begin{array}{l}
\left(\rho_{k}\right)_{j-1 / 2,+}^{n}=\left(\rho_{k}\right)_{j}^{n} \\
\left(U_{k}\right)_{j-1 / 2,+}^{n}=\left(U_{k}\right)_{j}^{n}
\end{array}\right.
$$

and

$$
\left(s_{k}\right)_{j-1 / 2,+}^{n}=\left(s_{k}\right)_{j}^{n} .
$$

Inserting previous results (B.26), (B.28), (B.29) and (B.30) in the scheme $W B R$ implies (for all $i$ ):

$$
\left\{\begin{array}{l}
\left(m_{k}\right)_{i}^{n+1}=\left(m_{k}\right)_{i}^{n} \\
\left(m_{k} U_{k}\right)_{i}^{n+1}=\left(m_{k} U_{k}\right)_{i}^{n} \\
\left(E_{k}\right)_{i}^{n+1}=\left(E_{k}\right)_{i}^{n}
\end{array}\right.
$$

Hence $W B R$ complies with Property 7.

\section{B.4. Property 8}

B.4.1. R scheme

Starting with (4.3) we may rewrite it as:

$$
\begin{aligned}
\left(\alpha_{2}\right)_{i}^{n+1}= & \left(1-\frac{\Delta t^{n}}{2 h_{i}}\left(r_{i+1 / 2}^{n}+r_{i-1 / 2}^{n}\right)\right)\left(\alpha_{2}\right)_{i}^{n}+\frac{\Delta t^{n}}{2 h_{i}}\left(r_{i+1 / 2}^{n}-\left(V_{I}\right)_{i}^{n}\right)\left(\alpha_{2}\right)_{i+1}^{n} \\
& +\frac{\Delta t^{n}}{2 h_{i}}\left(r_{i-1 / 2}^{n}+\left(V_{I}\right)_{i}^{n}\right)\left(\alpha_{2}\right)_{i-1}^{n} .
\end{aligned}
$$

If the following CFL constraint holds:

$$
\frac{\Delta t^{n}}{2 h_{i}}\left(r_{i+1 / 2}^{n}+r_{i-1 / 2}^{n}\right) \leq 1 \quad \forall i
$$


the Rusanov scheme $R$ obviously preserves positive values for $\alpha_{2}$. Moreover, noting that (B.32) remains unchanged when changing $\alpha_{2}$ into $1-\alpha_{2}$, we also conclude that $\alpha_{1}$ remains positive under the same CFL condition. Eventually, starting from (4.4) and using numerical flux definition (4.5), discrete values of $m_{k}$ comply with:

$$
\begin{aligned}
\left(m_{k}\right)_{i}^{n+1}=\left(1-\frac{\Delta t^{n}}{2 h_{i}}\left(r_{i+1 / 2}^{n}+r_{i-1 / 2}^{n}\right)\right)\left(m_{k}\right)_{i}^{n} & +\frac{\Delta t^{n}}{2 h_{i}} \frac{\epsilon_{i+1}}{\epsilon_{i}}\left(r_{i+1 / 2}^{n}-\left(U_{k}\right)_{i+1}^{n}\right)\left(m_{k}\right)_{i+1}^{n} \\
& +\frac{\Delta t^{n}}{2 h_{i}} \frac{\epsilon_{i-1}}{\epsilon_{i}}\left(r_{i-1 / 2}^{n}+\left(U_{k}\right)_{i-1}^{n}\right)\left(m_{k}\right)_{i-1}^{n} .
\end{aligned}
$$

Since $\left(m_{k}\right)_{i}^{n}>0$ for all $i$, partial masses also remain positive provided that the CFL condition (B.33) holds.

\section{B.4.2. MR scheme}

We assume here that: $(\hat{\epsilon})_{i+1 / 2}=\max \left(\epsilon_{i}, \epsilon_{i+1}\right)$. Following (4.7), the governing equation for the void fraction $\alpha_{2}$ in scheme $M R$ reads:

$$
\begin{aligned}
\left(\alpha_{2}\right)_{i}^{n+1}= & \left(1-\frac{\Delta t^{n}}{2 h_{i}}\left(\frac{(\hat{\epsilon})_{i+1 / 2}}{\epsilon_{i}} r_{i+1 / 2}^{n}+\frac{(\hat{\epsilon})_{i-1 / 2}}{\epsilon_{i}} r_{i-1 / 2}^{n}\right)\right)\left(\alpha_{2}\right)_{i}^{n}+\frac{\Delta t^{n}}{2 h_{i}}\left(\frac{(\hat{\epsilon})_{i+1 / 2}}{\epsilon_{i}} r_{i+1 / 2}^{n}-\left(V_{I}\right)_{i}^{n}\right)\left(\alpha_{2}\right)_{i+1}^{n} \\
& +\frac{\Delta t^{n}}{2 h_{i}}\left(\frac{(\hat{\epsilon})_{i-1 / 2}}{\epsilon_{i}} r_{i-1 / 2}^{n}+\left(V_{I}\right)_{i}^{n}\right)\left(\alpha_{2}\right)_{i-1}^{n}
\end{aligned}
$$

and the counterpart for the partial mass $m_{k}$ is:

$$
\begin{aligned}
\left(m_{k}\right)_{i}^{n+1}= & \left(1-\frac{\Delta t^{n}}{2 h_{i}}\left(\frac{(\hat{\epsilon})_{i+1 / 2}}{\epsilon_{i}} r_{i+1 / 2}^{n}+\frac{(\hat{\epsilon})_{i-1 / 2}}{\epsilon_{i}} r_{i-1 / 2}^{n}\right)\right)\left(m_{k}\right)_{i}^{n} \\
& +\frac{\Delta t^{n}}{2 h_{i}} \frac{(\epsilon)_{i+1}}{(\epsilon)_{i}}\left(\frac{(\hat{\epsilon})_{i+1 / 2}}{\epsilon_{i+1}} r_{i+1 / 2}^{n}-\left(U_{k}\right)_{i+1}^{n}\right)\left(m_{k}\right)_{i+1}^{n} \\
& +\frac{\Delta t^{n}}{2 h_{i}} \frac{(\epsilon)_{i-1}}{(\epsilon)_{i}}\left(\frac{(\hat{\epsilon})_{i-1 / 2}}{\epsilon_{i-1}} r_{i-1 / 2}^{n}+\left(U_{k}\right)_{i-1}^{n}\right)\left(m_{k}\right)_{i-1}^{n}
\end{aligned}
$$

Owing to inequalities $1 \leq \frac{(\hat{\epsilon})_{i+1 / 2}}{\epsilon_{i+1}}$ and $1 \leq \frac{(\hat{\epsilon})_{i+1 / 2}}{\epsilon_{i}}$, the CFL condition:

$$
\frac{\Delta t^{n}}{2 h_{i}}\left(\frac{(\hat{\epsilon})_{i+1 / 2}}{\epsilon_{i}} r_{i+1 / 2}^{n}+\frac{(\hat{\epsilon})_{i-1 / 2}}{\epsilon_{i}} r_{i-1 / 2}^{n}\right) \leq 1 \quad \forall i
$$

guarantees that both $\left(\alpha_{2}\right)_{i}^{n+1}$ and $\left(m_{k}\right)_{i}^{n+1}$ remain positive, assuming that associated initial values are positive. A similar remark as made above enables to conclude that $\left(\alpha_{2}\right)_{i}^{n+1} \leq 1$.

\section{B.4.3. WBR scheme}

We recall first that $\max \left(\left|\left(U_{k}\right)_{i+1 / 2,-}^{n}\right|,\left|\left(U_{k}\right)_{i+1 / 2,+}^{n}\right|\right) \leq\left(r_{W B}\right)_{i+1 / 2}^{n}$. The governing equation for the discrete values of $\alpha_{2}$ is exactly the same as the one occurring in the $R$ scheme. Thus, the CFL condition is the same as above and reads:

$$
\frac{\Delta t^{n}}{2 h_{i}}\left(\left(r_{W B}\right)_{i+1 / 2}^{n}+\left(r_{W B}\right)_{i-1 / 2}^{n}\right) \leq 1 \quad \forall i
$$

We turn now to the discrete partial masses. Starting with (4.11) and using numerical fluxes of $W B R$ as defined in (4.12), $m_{k}$ satisfies:

$$
\begin{array}{r}
\left(m_{k}\right)_{i}^{n+1}=\left(1-\frac{\Delta t^{n}}{2 h_{i}}\left(\left(r_{W B}\right)_{i+1 / 2}^{n}+\left(r_{W B}\right)_{i-1 / 2}^{n}\right)\right)\left(m_{k}\right)_{i}^{n}+\frac{\Delta t^{n}}{2 h_{i}}\left(\left(r_{W B}\right)_{i+1 / 2}^{n}-\left(U_{k}\right)_{i+1 / 2,-}^{n}\right)\left(\alpha_{k}\right)_{i+1}^{n}\left(\rho_{k}\right)_{i+1 / 2,-}^{n} \\
+\frac{\Delta t^{n}}{2 h_{i}}\left(\left(r_{W B}\right)_{i-1 / 2}^{n}+\left(U_{k}\right)_{i-1 / 2,+}^{n}\right)\left(\alpha_{k}\right)_{i-1}^{n}\left(\rho_{k}\right)_{i-1 / 2,+}^{n} \quad(\mathrm{~B} .39)
\end{array}
$$


Owing to the definition of $\left(r_{W B}\right)_{i+1 / 2}^{n},\left(m_{k}\right)_{i}^{n+1}$ is clearly a convex combination of the discrete partial mass $\left(m_{k}\right)_{i}^{n}$, and discrete densities $\left(\rho_{k}\right)_{i+1 / 2,-}^{n}$ and $\left(\rho_{k}\right)_{i-1 / 2,+}^{n}$, which are positive, provided that:

$$
\frac{\Delta t^{n}}{2 h_{i}}\left(\left(r_{W B}\right)_{i+1 / 2}^{n}+\left(r_{W B}\right)_{i-1 / 2}^{n}\right) \leq 1 .
$$

\section{Computing the interface states of $W B R$ scheme}

We first provide below the main guidelines to compute the interface state $Z_{i+1 / 2,-}^{n}$, assuming of course that $\epsilon_{i} \epsilon_{i+1} \neq 0$. Components of $Z_{i+1 / 2,-}^{n}$ should agree with:

$$
\operatorname{Inv}_{m}^{0}\left(Z_{i+1 / 2,-}^{n}, \epsilon_{i}\right)=\operatorname{Inv}_{m}^{0}\left(Z_{i+1}^{n}, \epsilon_{i+1}\right) .
$$

As a consequence, we have $\left(\alpha_{k}\right)_{i+1 / 2,-}^{n}=\left(\alpha_{k}\right)_{i+1}^{n},\left(s_{k}\right)_{i+1 / 2,-}^{n}=\left(s_{k}\right)_{i+1}^{n}$ and:

$$
\begin{gathered}
\epsilon_{i}\left(\alpha_{k}\right)_{i+1 / 2,-}^{n}\left(\rho_{k}\right)_{i+1 / 2,-}^{n}\left(U_{k}\right)_{i+1 / 2,-}^{n}=\epsilon_{i+1}\left(\alpha_{k}\right)_{i+1}^{n}\left(\rho_{k}\right)_{i+1}^{n}\left(U_{k}\right)_{i+1}^{n} \\
2 h_{k}\left(\left(s_{k}\right)_{i+1 / 2,-}^{n},\left(\rho_{k}\right)_{i+1 / 2,-}^{n}\right)+\left(\left(U_{k}\right)_{i+1 / 2,-}^{n}\right)^{2}=2 h_{k}\left(\left(s_{k}\right)_{i+1}^{n},\left(\rho_{k}\right)_{i+1}^{n}\right)+\left(\left(U_{k}\right)_{i+1}^{n}\right)^{2}
\end{gathered}
$$

where: $\rho_{k} h_{k}\left(s_{k}, \rho_{k}\right)=\rho_{k} e_{k}\left(P_{k}\left(s_{k}, \rho_{k}\right), \rho_{k}\right)+P_{k}\left(s_{k}, \rho_{k}\right)$. Setting as the main unknown $X=\left(\rho_{k}\right)_{i+1 / 2,-}^{n}$, and substituting in the last equation, one needs to solve:

$$
\psi_{i+1 / 2,-}^{n}(X)=0
$$

where:

$$
\psi_{i+1 / 2,-}^{n}(X) \stackrel{\text { def }}{=} 2\left(h_{k}\left(\left(s_{k}\right)_{i+1}^{n}, X\right)-h_{k}\left(\left(s_{k}\right)_{i+1}^{n},\left(\rho_{k}\right)_{i+1}^{n}\right)\right)+\left(\left(U_{k}\right)_{i+1}^{n}\right)^{2}\left(\frac{\left(\epsilon_{i+1}\left(\rho_{k}\right)_{i+1}^{n}\right)^{2}}{\left(\epsilon_{i} X\right)^{2}}-1\right) .
$$

The solution is obviously $X=\left(\rho_{k}\right)_{i+1}^{n}$ when $\epsilon_{i}=\epsilon_{i+1}$. If $\epsilon_{i} \neq \epsilon_{i+1}$, we proceed as follows:

- If $\left(U_{k}\right)_{i+1}^{n}=0$, the solution of (C.1) is $X=\left(\rho_{k}\right)_{i+1}^{n}$.

- Otherwise, one first computes $X_{\min }>0$ solution of:

$$
X_{\min }^{3}\left(\partial_{\rho_{k}}\left(h_{k}\left(s_{k}, \rho_{k}\right)\right)\right)\left(\left(s_{k}\right)_{i+1}^{n}, X_{\min }\right)=\left(\frac{\left(U_{k}\right)_{i+1}^{n} \epsilon_{i+1}\left(\rho_{k}\right)_{i+1}^{n}}{\epsilon_{i}}\right)^{2}>0 .
$$

The following two possibilities arise:

- Either:

$$
\psi_{i+1 / 2,-}^{n}\left(X_{\min }\right)>0 .
$$

In that case, (C.1) has no solution. The computation is stopped. Another alternative consists in setting the solution to $X_{\min }$.

- Otherwise, the equation (C.1) admits two solutions $\left.\left.X^{-} \in\right] 0, X_{\min }\right]$ and $X^{+} \in\left[X_{\min },+\infty[\right.$. The final solution is $X=X^{-}$if $\left(\rho_{k}\right)_{i+1}^{n} \leq X_{\min }$, and $X=X^{+}$if $X_{\min } \leq\left(\rho_{k}\right)_{i+1}^{n}$.

$Z_{i+1 / 2,+}^{n}$ is obtained with a similar way, using $\left(\rho_{k}\right)_{i}^{n}$ to determine the admissible branch of solutions.

Acknowledgements. We would like to thank Frédéric Archambeau and Thierry Gallouët who kindly accepted to read the initial version of the manuscript, which helped much. This work has been achieved in the framework of the NEPTUNE project, with financial support from CEA (Commissariat à l'Énergie Atomique), EDF, IRSN (Institut de Radioprotection 
et Sûreté Nucléaire) and AREVA-NP. Part of the financial support of the first author is provided by ANRT (Association Nationale de la Recherche Technique, Ministère de la Recherche) through a EDF/CIFRE contract, and also by FSE (Fonds Social Européen). All computational facilities were provided by EDF.

\section{REFERENCES}

[1] A. Ambroso, C. Chalons, F. Coquel, E. Godlewski, F. Lagoutière, P.A. Raviart and N. Seguin, Working group on the interfacial coupling of models. http://www.ann.jussieu.fr/groupes/cea (2003).

[2] N. Andrianov and G. Warnecke, The Riemann problem for the Baer-Nunziato two-phase flow model. J. Comput. Phys. 195 (2004) 434-464.

[3] E. Audusse, F. Bouchut, M.O. Bristeau, R. Klein and B. Perthame, A fast and stable well-balanced scheme with hydrodynamic reconstruction for shallow water flows. SIAM J. Sci. Comput. 25 (2004) 2050-2065.

[4] M.R. Baer and J.W. Nunziato, A two-phase mixture theory for the deflagration to detonation transition (DDT) in reactive granular materials. Int. J. Multiphase Flow 12 (1986) 861-889.

[5] F. Bouchut, Nonlinear stability of Finite Volume methods for hyperbolic conservation laws, and well-balanced schemes for sources, Frontiers in Mathematics series. Birkhauser (2004).

[6] B. Boutin, F. Coquel and E. Godlewski, Dafermos Regularization for Interface Coupling of Conservation Laws, in Hyperbolic problems: Theory, Numerics, Applications, Springer (2008) 567-575.

[7] T. Buffard, T. Gallouët and J.-M. Hérard, A sequel to a rough Godunov scheme. Application to real gases. Comput. Fluids 29 (2000) 813-847.

[8] A. Chinnayya, A.Y. Le Roux and N. Seguin, A well-balanced numerical scheme for shallow-water equations with topography: the resonance phenomena. Int. J. Finite Volumes 1 (2004) available at http://www.latp.univ-mrs.fr/IJFV/.

[9] F. Coquel, T. Gallouët, J.M. Hérard and N. Seguin, Closure laws for a two-fluid two-pressure model. C. R. Acad. Sci. Paris. I-332 (2002) 927-932.

[10] R. Eymard, T. Gallouët and R. Herbin, Finite Volume methods, in Handbook of Numerical Analysis VII, P.G. Ciarlet and J.L. Lions Eds., North Holland (2000) 715-1022.

[11] T. Gallouët, J.-M. Hérard and N. Seguin, A hybrid scheme to compute contact discontinuities in one dimensional Euler systems. ESAIM: M2AN 36 (2002) 1133-1159.

[12] T. Gallouët, J.-M. Hérard and N. Seguin, Some recent Finite Volume schemes to compute Euler equations using real gas EOS. Int. J. Num. Meth. Fluids 39 (2002) 1073-1138.

[13] T. Gallouët, J.-M. Hérard and N. Seguin, Some approximate Godunov schemes to compute shallow water equations with topography. Comput. Fluids 32 (2003) 479-513.

[14] T. Gallouët, J.-M. Hérard and N. Seguin, Numerical modelling of two phase flows using the two-fluid two-pressure approach. Math. Mod. Meth. Appl. Sci. 14 (2004) 663-700.

[15] L. Girault and J.-M. Hérard, Multidimensional computations of a two-fluid hyperbolic model in a porous medium. AIAA paper 2009-3540 (2009) available at http://www. aiaa.org.

[16] P. Goatin and P. Le Floch, The Riemann problem for a class of resonant hyperbolic systems of balance laws. Ann. Inst. Henri Poincaré, Anal. non linéaire 21 (2004) 881-902.

[17] E. Godlewski, Coupling fluid models. Exploring some features of interfacial coupling, in Proceedings of Finite Volumes for Complex Applications V, Aussois, France, June 8-13 (2008).

[18] E. Godlewski and P.A. Raviart, The numerical interface coupling of nonlinear hyperbolic systems of conservation laws: 1 . The scalar case. Numer. Math. 97 (2004) 81-130.

[19] E. Godlewski, K.C. Le Thanh and P.-A. Raviart, The numerical interface coupling of nonlinear hyperbolic systems of conservation laws: II. The case of systems. ESAIM: M2AN 39 (2005) 649-692.

[20] S.K. Godunov, Finite difference method for numerical computation of discontinuous solutions of the equations of fluid dynamics. Mat. Sb. 47 (1959) 271-300.

[21] J.M. Greenberg and A.Y. Leroux, A well balanced scheme for the numerical processing of source terms in hyperbolic equations. SIAM J. Numer. Anal. 33 (1996) 1-16.

[22] V. Guillemaud, Modélisation et simulation numérique des écoulements diphasiques par une approche bifluide à deux pressions. Ph.D. Thesis, Université Aix Marseille I, Marseille, France (2007).

[23] P. Helluy, J.-M. Hérard and H. Mathis, A well-balanced approximate Riemann solver for variable cross-section compressible flows. AIAA paper 2009-3888 (2009) available at http://www.aiaa.org.

[24] J.M. Hérard, A rough scheme to couple free and porous media. Int. J. Finite Volumes 3 (2006) available at http://www. latp. univ-mrs.fr/IJFV/.

[25] J.-M. Hérard, A three-phase flow model. Math. Comp. Model. 45 (2007) 432-455.

[26] J.-M. Hérard, Un modèle hyperbolique diphasique bi-fluide en milieu poreux. C. r., Méc. 336 (2008) 650-655.

[27] A.K. Kapila, S.F. Son, J.B. Bdzil, R. Menikoff and D.S. Stewart, Two-phase modeling of a DDT: structure of the velocity relaxation zone. Phys. Fluids 9 (1997) 3885-3897. 
[28] D. Kröner and M.D. Thanh, Numerical solution to compressible flows in a nozzle with variable cross-section. SIAM J. Numer. Anal. 43 (2006) 796-824.

[29] D. Kröner, P. Le Floch and M.D. Thanh, The minimum entropy principle for compressible fluid flows in a nozzle with discontinuous cross-section. ESAIM: M2AN 42 (2008) 425-442.

[30] C.A. Lowe, Two-phase shock-tube problems and numerical methods of solution. J. Comput. Phys. 204 (2005) $598-632$.

[31] D.W. Schwendeman, C.W. Wahle and A.K. Kapila, The Riemann problem and a high resolution Godunov method for a model of compressible two-phase flow. J. Comput. Phys. 212 (2006) 490-526. 